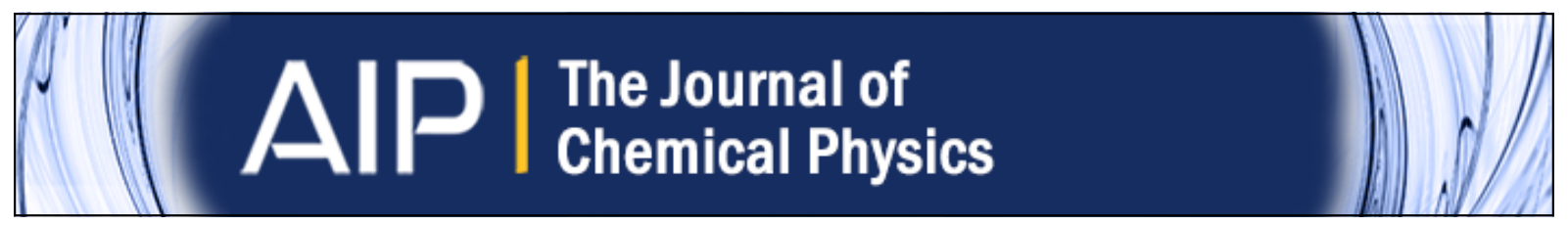

Ab initio analytical Raman intensities for periodic systems through a coupled perturbed Hartree-Fock/Kohn-Sham method in an atomic orbital basis. I. Theory Lorenzo Maschio, Bernard Kirtman, Michel Rérat, Roberto Orlando, and Roberto Dovesi

Citation: The Journal of Chemical Physics 139, 164101 (2013); doi: 10.1063/1.4824442

View online: http://dx.doi.org/10.1063/1.4824442

View Table of Contents: http://scitation.aip.org/content/aip/journal/jcp/139/16?ver=pdfcov

Published by the AIP Publishing

Advertisement:

AlP Re-register for Table of Content Alerts 


\title{
Ab initio analytical Raman intensities for periodic systems through a coupled perturbed Hartree-Fock/Kohn-Sham method in an atomic orbital basis. I. Theory
}

\author{
Lorenzo Maschio, ${ }^{1, a)}$ Bernard Kirtman, ${ }^{2}$ Michel Rérat, ${ }^{3}$ Roberto Orlando, ${ }^{1}$ \\ and Roberto Dovesi ${ }^{1}$ \\ ${ }^{1}$ Dipartimento di Chimica, Università di Torino and NIS-Nanostructured Interfaces and Surfaces-Centre of \\ Excellence, Via P. Giuria 7, 10125 Torino, Italy \\ ${ }^{2}$ Department of Chemistry and Biochemistry, University of California, Santa Barbara, California 93106, USA \\ ${ }^{3}$ Equipe de Chimie Physique, IPREM UMR5254, Université de Pau et des Pays de l'Adour, 64000 Pau, France
}

(Received 16 August 2013; accepted 20 September 2013; published online 22 October 2013)

\begin{abstract}
We present a fully analytical formulation for calculating Raman intensities of crystalline periodic systems using a local basis set. Numerical differentiation with respect to atomic coordinates and with respect to wavevectors is entirely avoided as is the determination of crystal orbital coefficient derivatives with respect to nuclear displacements. Instead, our method utilizes the orbital energy-weighted density matrix and is based on the self-consistent solution of first- and second-order Coupled Perturbed Hartree-Fock/Kohn-Sham equations for the electronic response to external electric fields at the equilibrium geometry. This method has also been implemented in the CRYSTAL program, which uses a Gaussian type basis set. ( 2013 AIP Publishing LLC. [http://dx.doi.org/10.1063/1.4824442]
\end{abstract}

\section{INTRODUCTION}

Raman spectroscopy undoubtedly ranks among the favorite techniques for the characterization of crystalline materials. However, despite the relative simplicity of the experimental setup and the wide availability of high-quality instrumentation, there remain difficulties associated with the determination of absolute and relative intensities across the entire frequency range. ${ }^{1}$ This is mainly due to the absence of a reference channel to compensate for instrumental sensitivity variation with time and/or wavelength. In this connection, the ability to obtain intensities from ab initio calculations is highly important not only for predictive purposes but also to support the analysis of experimental data and to help to compare spectra from different instruments.

Within the Placzek approximation, ${ }^{2}$ the key properties involved in computing nonresonant Raman susceptibilities are the partial derivatives of the polarizability tensor with respect to atomic positions:

$$
\left.\frac{\partial \alpha_{b, c}}{\partial \mathcal{R}_{a}^{A}}\right|_{\mathcal{R}_{0}}=\left.\frac{\partial^{3} E^{T O T}}{\partial \mathcal{R}_{a}^{A} \partial \mathcal{E}_{b} \partial \mathcal{E}_{c}}\right|_{\mathcal{E}=0, \mathcal{R}_{0}} .
$$

Here $\overrightarrow{\mathcal{R}}^{A}$ is the coordinate of atom $A, \overrightarrow{\mathcal{E}}$ is the electric field and $a, b, c$ indicate cartesian directions.

While ab initio methods for the evaluation of these quantities were developed for molecules in the early $1980 \mathrm{~s},{ }^{3,4}$ fundamental problems arise when trying to formulate a computable expression for periodic crystalline systems. These problems have delayed solid state applications.

In order to appreciate the modifications necessary to convert from the treatment for a molecule to that of an infinite periodic system, we recall here that the expression for the

\footnotetext{
a)Electronic mail: lorenzo.maschio@unito.it
}

scalar dipole interaction between an electron in a molecule and a uniform static electric field $\overrightarrow{\mathcal{E}}$ (electron charge unit: $e=-1$ a.u.),

$$
\hat{V}(\vec{r})=\overrightarrow{\mathcal{E}} \cdot \vec{r}
$$

is not consistent with the periodic boundary conditions for an infinite system, because it is unbound and breaks translational invariance. Solutions to this problem have been proposed by several authors. ${ }^{5-12}$ One common approach relies on an alternative formulation of the electronic interaction operator which, in the Bloch basis, is diagonal with respect to the reciprocal space $\vec{k}$ vector. Provided the system has a non-zero bandgap, this operator may be written as

$$
\hat{\Omega}(\vec{k})=\imath \overrightarrow{\mathcal{E}} \cdot e^{i \vec{k} \cdot \vec{r}} \vec{\nabla}_{k} e^{-l \vec{k} \cdot \vec{r}}=\sum_{b} \mathcal{E}_{b} \hat{\Omega}_{b}(\vec{k}) .
$$

The evaluation of $\hat{\Omega}$ requires a derivative with respect to $\vec{k}$. Algorithms capable of dealing with this derivative have been implemented in plane wave codes ${ }^{13-16}$ although the role of crystal orbital phase factors is often ignored, as discussed in this paper and in Refs. 11 and 17. A rather different approach, appropriate for an atomic orbital (AO) basis, is employed herein.

One possible way of proceeding is to treat the interaction term in (3) perturbatively. Then, the wavefunction and electric dipole properties can be obtained, for example, through a periodic Coupled-perturbed Hartree-Fock (CPHF) or KohnSham (CPKS) approach. Such a CPHF/CPKS treatment has recently been developed ${ }^{18}$ and implemented, ${ }^{19,20}$ by some of the present authors, in the CRYSTAL09 program, that uses a local Gaussian basis set. ${ }^{21,22}$

Periodic codes that allow one to compute Raman intensities mainly adopt a density functional theory (DFT) CPKS approach together with a plane wave basis set. Differentiation with respect to nuclear coordinates is, then, carried out either 
numerically ${ }^{23-26}$ or analytically. ${ }^{27-30}$ To our knowledge, a formalism in a local basis, such as we report in this work, has not been previously presented. In contrast with previous treatments (except for Ref. 28) we also avoid the time-consuming calculation of derivatives of orbital coefficients with respect to nuclear displacements. In addition, the role of exact exchange has generally not been considered, thereby precluding the use of hybrid functionals in the CPKS treatment. Finally, in several cases the derivative with respect to $\vec{k}$ is treated numerically (e.g., by means of Berry phase techniques), rather than analytically, as done here.

We have recently presented an analytical approach for the evaluation of IR intensities. ${ }^{17,31}$ It combines the analytical periodic $\mathrm{CPHF} / \mathrm{CPKS}$ treatment of electric field derivatives with analytical gradients (i.e., geometric first derivatives) of the total energy. ${ }^{36}$ Gradients of the wavefunction are avoided (following the $n+1$ rule $^{32}$ ) through the use of the orbital-energy weighted density matrix. This was demonstrated to be efficient and numerically stable, when combined with the usual numerical integrations over reciprocal space.

In the current work, we extend the formalism to Raman intensities. Again, our treatment avoids calculating gradients of the wavefunction (second derivatives with respect to the electric fields are required instead) as opposed to the corresponding treatment for molecules presented by Quinet and Champagne. ${ }^{33}$ Our procedure is fully analytical except, of course, for the integrations over reciprocal space. Moreover, we develop further a simple way of treating the derivatives with respect to the wave vector introduced in earlier papers. ${ }^{17-19}$ The numerical validation and performance, as implemented in CRYSTAL, is discussed in Paper II. ${ }^{34}$

The overall result is the first implementation of a fully analytical approach for computing Raman intensities in a Gaussian-type basis that is routinely usable for periodic systems of any dimensionality, allowing also for the use of hybrid functionals. Readers who are not interested in following the entire derivation in detail, will find the implemented expression for the Raman intensities given by Eq. (77).

This paper is organized as follows: in Secs. II A and II B the CPHF theory preliminary to the present development is briefly reviewed. In Sec. II C, the treatment of the perturbation due to atomic displacements is described. Then, starting from the mixed second derivative of the total energy with respect to the atomic displacement and the electric field (Sec. II D), we derive the fully analytical expression for the Raman tensor in Sec. III. In Sec. III E, the extension to CPKS theory is described. Finally, in Sec. IV conclusions are drawn and some future work is noted.

\section{CPHF THEORY AND PRELIMINARIES}

Let us briefly introduce the notation, adopted in the following:

- Greek indices $\mu, v, \ldots$ will label $\mathrm{AO}$ basis functions in the unit cell.

- Direct space lattice vectors will be indicated as $\vec{g}, \vec{h}, \vec{n} \ldots$
- $k$-vectors of the Brillouin Zone (BZ) sampling in reciprocal space will be indicated as $\vec{k}$.

- A general cartesian direction (x,y,z) will be expressed by lower case letters $a, b, \ldots$.

- Matrices denoted as $S, F, D, \ldots$ represent direct space quantities, while reciprocal space quantities are denoted as $S(\vec{k}), F(\vec{k}), D(\vec{k}), \ldots$

- Direct space and reciprocal space quantities are connected through standard Fourier and back-Fourier transforms, $\mathcal{F}$ and $\overline{\mathcal{F}}$, respectively,

$$
\begin{aligned}
S(\vec{k}) & =\mathcal{F}[S], \\
S & =\overline{\mathcal{F}}[S(\vec{k})] .
\end{aligned}
$$

\section{A. Unit cell total energy for periodic systems}

We start with the HF expression for the total energy of a periodic system in the presence of an electric field

$$
E^{T O T}=\frac{1}{2} \operatorname{Tr}((H+F) D)+E^{N N}-\sum_{A, b} \mathcal{E}_{b} Z_{A} \mathcal{R}_{b}^{A},
$$

where $F$ is the electronic Fock matrix made of a one-electron term $H$ and two-electron terms

$$
\begin{gathered}
F=H+(B+T+S q), \\
H=h+\sum_{b} \mathcal{E}_{b} \Omega_{b} .
\end{gathered}
$$

In this expression, which has been adapted from Refs. 35 and 36 , Tr signifies the trace and the quantities $D, S, h, B, T$, and $\Omega$ are the direct space AO matrices defined below:

- $D$ is the density matrix obtained by means of a Fourier back-transform

$$
D=\overline{\mathcal{F}}[D(\vec{k})]
$$

of the corresponding $k$-space matrix $D(\vec{k})$ calculated as (assuming closed shells)

$$
D(\vec{k})=C(\vec{k}) n C^{\dagger}(\vec{k}),
$$

where $C$ is the matrix of crystal orbital eigenvectors (see Eq. (12) below) and $n$ is the diagonal occupation number matrix with eigenvalues $=2$ for occupied orbitals and $=0$ for virtual orbitals.

- $S$ is the AO overlap matrix.

- $h$ is the core hamiltonian matrix containing the kinetic energy and nuclear-electron interaction terms.

- $B$ is the electron-electron interaction term. Its matrix elements are given by

$$
B_{\mu, \nu}^{\vec{g}}=\sum_{\rho, \tau, h, n} D_{\rho \tau \vec{n}}\left(\mu \nu^{\vec{g}}|| \rho^{\vec{h}} \tau^{\vec{h}+\vec{n}}\right),
$$

where the bielectronic integrals in Eq. (9) are partly computed exactly and partly approximated by a bipolar expansion.

- $T$ represents the infinite summation contribution to the electron-electron interaction, which is not reported here. An explicit expression can be found in Saunders 
et al. ${ }^{35}$ We do note that $T$ depends upon the density matrix, i.e.,

$$
T=D \mathcal{T},
$$

where $\mathcal{T}$ is the interaction of a field integral with a set of point multipoles, and includes a potential function that depends upon the dimensionality, namely, the Coulomb potential in the molecular case, the EulerMacLaurin potential in 1D periodic systems, ${ }^{37,38}$ Parry's potential for 2D periodic systems, ${ }^{39,40}$ and the Ewald potential for 3D systems. ${ }^{35,41}$

- $\mathcal{R}_{a}^{A}$ is the cartesian coordinate of atom A along direction $a$.

- $Z_{A}$ is the nuclear charge of atom A.

- $E^{N N}$ is the nuclear-nuclear interaction energy.

- $\Omega_{b}$ is the AO matrix representation, in direct space, for the $b$ cartesian component of the $\hat{\Omega}(\vec{k})$ operator in Eq. (3). The properties of this matrix, which is central to our approach, will be discussed in detail in Sec. II B.

- $q$ is the value of the spheropole. This term arises only in $3 \mathrm{D}$ periodic systems. It occurs because the charge distribution is approximated by a model in the long range and is needed in order to guarantee that the average of the electrostatic potential vanishes. We note that $q$ depends upon the density matrix, i.e.,

$$
q=\operatorname{Tr}(D \mathcal{Q}),
$$

where $\mathcal{Q}$ is the matrix of spheropolar integrals whose explicit form can be found in Refs. 35 and 36.

\section{B. Perturbation treatment of an external electric field}

The periodic CPHF method as implemented in CRYSTAL has been reported in a number of papers. ${ }^{18-20,42}$ Thus, we provide here only a summary of the key relations employed in this work. The crystal orbital coefficients in Eq. (8) may be obtained by solving the Fock equation (derived from the energy expression)

$$
\begin{gathered}
F(\vec{k}) C(\vec{k})=S(\vec{k}) C(\vec{k}) \epsilon(\vec{k}), \\
F(\vec{k})=\mathcal{F}[F],
\end{gathered}
$$

where $\mathcal{F}$ is the Fourier transform operator. Equation (12) is solved subject to the normalization condition

$$
C^{\dagger}(\vec{k}) S(\vec{k}) C(\vec{k})=I,
$$

where $I$ is the identity matrix.

Here $\epsilon(\vec{k})$ is the matrix of Lagrange multipliers. We use a non-canonical formulation so that this matrix is only block diagonal in the occupied/virtual crystal orbital space, except at zero field (in which case it is fully diagonal).

The Fock equation is solved at each $\vec{k}$ point in the irreducible BZ to obtain the crystal orbital coefficients for a given field (usually, the spheropole term is removed from the $k$-space Fock equation ${ }^{17,35}$ and, subsequently, added to the total energy; however, for simplicity here it is left in the Fock matrix). We write $C(\vec{k})$ as a function of electric field using a Taylor series in the field components:

$$
\begin{aligned}
C(\vec{k})= & C^{(0)}(\vec{k})+\sum_{a=x, y, z} \mathcal{E}_{a} C^{\left(\mathcal{E}_{a}\right)}(\vec{k}) \\
& +\sum_{a, b=x, y, z} \mathcal{E}_{a} \mathcal{E}_{b} C^{\left(\mathcal{E}_{a}, \mathcal{E}_{b}\right)}(\vec{k})+\cdots
\end{aligned}
$$

Then, the first derivative of the density matrix Eq. (8) with respect to the field is

$$
D^{\left(\mathcal{E}_{a}\right)}(\vec{k})=C^{\left(\mathcal{E}_{a}\right)}(\vec{k}) n C^{(0) \dagger}(\vec{k})+C^{(0)}(\vec{k}) n C^{\left(\mathcal{E}_{a}\right) \dagger}(\vec{k}),
$$

whereas, the corresponding electric field derivative of the normalization condition (Eq. (14)) is

$$
C^{\left(\mathcal{E}_{a}\right) \dagger}(\vec{k}) S(\vec{k}) C^{(0)}(\vec{k})+C^{(0) \dagger}(\vec{k}) S(\vec{k}) C^{\left(\mathcal{E}_{a}\right)}(\vec{k})=0 .
$$

The second derivative of the density matrix Eq. (8) with respect to the field may be expressed as

$$
\begin{aligned}
D^{\left(\mathcal{E}_{a}, \mathcal{E}_{b}\right)}(\vec{k})= & C^{\left(\mathcal{E}_{a}, \mathcal{E}_{b}\right)}(\vec{k}) n C^{(0) \dagger}(\vec{k}) \\
& +P_{a, b} C^{\left(\mathcal{E}_{a}\right)}(\vec{k}) n C^{\left(\mathcal{E}_{b}\right) \dagger}(\vec{k}) \\
& +C^{(0)}(\vec{k}) n C^{\left(\mathcal{E}_{a}, \mathcal{E}_{b}\right) \dagger}(\vec{k}),
\end{aligned}
$$

where $P_{a, b}$ is the permutation operator; the corresponding derivative of the normalization condition (Eq. (14)) is given by

$$
\begin{aligned}
& C^{\left(\mathcal{E}_{a}, \mathcal{E}_{b}\right) \dagger}(\vec{k}) S(\vec{k}) C^{(0)}(\vec{k})+P_{a, b} C^{\left(\mathcal{E}_{a}\right) \dagger}(\vec{k}) S(\vec{k}) C^{\left(\mathcal{E}_{b}\right)}(\vec{k}) \\
& \quad+C^{(0) \dagger}(\vec{k}) S(\vec{k}) C^{\left(\mathcal{E}_{a}, \mathcal{E}_{b}\right)}(\vec{k})=0 .
\end{aligned}
$$

In order to develop an $\mathrm{AO}$ matrix representation of $\hat{\Omega}(\vec{k})$ we start by operating on an arbitrary $\mathrm{CO}\left|\phi_{i}\right\rangle=\sum_{\nu}|\nu\rangle C_{v}, i$ and projecting on the left-hand side with the $\mathrm{AO}$ basis function $\langle\mu|$. This yields the mixed $(\mathrm{AO} / \mathrm{CO})$ relation

$$
\begin{aligned}
& \sum_{\nu}\left\langle\mu\left|\hat{\Omega}_{b}(\vec{k})\right| \nu\right\rangle C_{\nu, i}(\vec{k}) \\
& \quad=\sum_{\nu}\left[\left(Z_{b}\right)_{\mu \nu}(\vec{k})+i S_{\mu \nu}^{\left(k_{b}\right)}(\vec{k})+i S_{\mu \nu}(\vec{k}) \frac{\partial}{\partial k_{b}}\right] C_{\nu, i}(\vec{k}) .
\end{aligned}
$$

Here $Z_{b}(\vec{k})$ is the AO matrix that represents the component of the electronic position coordinate along $b$ and $S^{\left(k_{b}\right)}(\vec{k})$ is the derivative of the AO overlap matrix, $S$, with respect to the wave vector $k_{b}$. This last relation, such as Eqs. (12)-(14), is valid at any arbitrary field and geometry.

It is useful to define the derivative of the zero field coefficients with respect to vector $\vec{k}$ in terms of the $\mathrm{CO}$ matrix $Q_{b}(\vec{k})$ given by

$$
\frac{\partial}{\partial k_{b}} C^{(0)}(\vec{k})=C^{(0)}(\vec{k}) Q_{b}(\vec{k}) .
$$

The diagonal elements of $\mathrm{Q}$ contain an undetermined imaginary component (which reflects an arbitrary phase factor in the CO coefficients). ${ }^{11,43,44}$ This imaginary component, in turn, causes the dipole moment to be determined only up to the addition of an arbitrary lattice vector. In our previous work (Refs. 17 and 31), we showed that the IR intensities are unaffected by this indeterminacy as long as the nuclear motion 
does not involve a change in lattice parameters. Since it is known that polarizabilities are independent of the imaginary diagonal components of Q (Ref. 11) a similar result holds for Raman intensities and, in this case, for all nuclear motions.

Even after making the substitution of Eq. (21) into Eq. (20) the last term on the rhs does not have the desired form, i.e., $X C$, where $X$ is an AO matrix. This difficulty is solved $^{45}$ by multiplying on the right with the orthonormality condition (14) to give

$$
\imath S(\vec{k}) \frac{\partial}{\partial k_{b}} C(\vec{k})=\imath\left[S(\vec{k})\left(\frac{\partial C(\vec{k})}{\partial k_{b}}\right) C^{\dagger}(\vec{k}) S(\vec{k})\right] C(\vec{k}) .
$$

Again, the above equation is valid for arbitrary geometry and field. The quantity in square brackets is treated selfconsistently in solving the CPHF equations.

Finally, it is convenient to introduce the matrix

$$
M_{b}(\vec{k})=Z_{b}(\vec{k})+\imath S^{\left(k_{b}\right)}(\vec{k})
$$

which depends upon geometry but not field.

Then, combining Eqs. (20) and (22) with the definition

$$
\iota \Xi_{b}(\vec{k})=\imath S(\vec{k})\left(\frac{\partial}{\partial k_{b}} C(\vec{k})\right) C^{\dagger}(\vec{k}) S(\vec{k}),
$$

leads to an $\mathrm{AO}$ matrix expression for $\Omega_{b}(\vec{k})$, i.e.,

$$
\Omega_{b}(\vec{k})=\bar{M}_{b}(\vec{k})+\imath \bar{\Xi}_{b}(\vec{k}) .
$$

In Eq. (25) we have, for convenience, introduced the hermitized variants of $M_{b}(\vec{k})$ and $\Xi_{b}(\vec{k})$,

$$
\begin{aligned}
& \bar{M}_{b}(\vec{k})=\frac{1}{2}\left(M_{b}(\vec{k})+M_{b}^{\dagger}(\vec{k})\right), \\
& \iota \bar{\Xi}_{b}(\vec{k})=\frac{1}{2} l\left(\Xi_{b}(\vec{k})-\Xi_{b}^{\dagger}(\vec{k})\right),
\end{aligned}
$$

because these two quantities are not separately hermitian even though their sum $\Omega_{b}(\vec{k})$ is hermitian. This allows us to treat $M_{b}(\vec{k})$ and $\iota \Xi_{b}(\vec{k})$ separately in the following development.

For zero field we can exploit Eq. (21) to get

$$
\Omega_{b}^{(0)}(\vec{k})=\bar{M}_{b}(\vec{k})+\imath\left[S(\vec{k}) C^{(0)}(\vec{k}) \bar{Q}_{b}(\vec{k}) C^{(0) \dagger}(\vec{k}) S(\vec{k})\right],
$$

where, in analogy with Eq. (27), we have defined

$$
{ }_{\imath} \bar{Q}_{b}(\vec{k})=\frac{1}{2} l\left(Q_{b}(\vec{k})-Q_{b}^{\dagger}(\vec{k})\right) .
$$

This yields the energy contribution linear in the field (see Eq. (6)) as $\sum_{b} \mathcal{E}_{b} \operatorname{Tr}\left(D^{(0)} \Omega_{b}^{(0)}\right)$, with $\Omega_{b}^{(0)}$ defined as the backFourier transform of $\Omega_{b}^{(0)}(\vec{k})$,

$$
\Omega_{b}^{(0)}=\overline{\mathcal{F}}\left[\Omega_{b}^{(0)}(\vec{k})\right] .
$$

\section{First-order CPHF equations}

In order to obtain the first-order CPHF electric field equation for $C^{\left(\mathcal{E}_{a}\right)}$ we take the derivative of Eq. (12) with respect to the field. This leads to

$$
\begin{aligned}
& {\left[F^{\left(\mathcal{E}_{b}\right)}(\vec{k}) C^{(0)}(\vec{k})+F^{(0)}(\vec{k}) C^{\left(\mathcal{E}_{b}\right)}(\vec{k})\right]} \\
& \quad=S(\vec{k})\left[C^{\left(\mathcal{E}_{b}\right)}(\vec{k}) \epsilon^{(0)}(\vec{k})+C^{(0)}(\vec{k}) \epsilon^{\left(\mathcal{E}_{b}\right)}(\vec{k})\right],
\end{aligned}
$$

which holds for arbitrary geometry and zero field. Equation (31) is solved by writing

$$
C^{\left(\mathcal{E}_{b}\right)}(\vec{k})=C^{(0)}(\vec{k}) U^{\left(\mathcal{E}_{b}\right)}(\vec{k})
$$

and, then, determining $U^{\left(\mathcal{E}_{b}\right)}(\vec{k})$ self-consistently so as to satisfy the condition that the off-diagonal blocks of $\epsilon^{\left(\mathcal{E}_{b}\right)}(\vec{k})$ are zero. The diagonal blocks (occ-occ or virt-virt) of $U^{\left(\mathcal{E}_{b}\right)}(\vec{k})$ are arbitrary, apart from the normalization condition of Eq. (17), which is trivially satisfied in CRYSTAL ${ }^{19}$ by taking these blocks to be zero. This requires $U^{\left(\mathcal{E}_{b}\right)}(\vec{k})$ to be anti-hermitian. With that choice the diagonal blocks of the Lagrange multiplier matrix become

$$
\begin{aligned}
\epsilon^{\left(\mathcal{E}_{b}\right)}(\vec{k})= & G_{D}^{\left(\mathcal{E}_{b}\right)}(\vec{k}) \\
= & \left\{C^{(0) \dagger}(\vec{k})\left[B^{\left(\mathcal{E}_{b}\right)}(\vec{k})+T^{\left(\mathcal{E}_{b}\right)}(\vec{k})+q^{\left(\mathcal{E}_{b}\right)} S(\vec{k})+\bar{M}_{b}(\vec{k})\right]\right. \\
& \left.\times C^{(0)}(\vec{k})+\imath \bar{Q}_{b}(\vec{k})\right\}_{D}
\end{aligned}
$$

as indicated by the subscript $D$.

As already done in earlier papers, ${ }^{18}$ we have introduced here the shorthand notation

$$
G^{\left(\mathcal{E}_{b}\right)}(\vec{k})=C^{(0) \dagger}(\vec{k}) F^{\left(\mathcal{E}_{b}\right)}(\vec{k}) C^{(0)}(\vec{k})
$$

for the quantity in curly brackets in Eq. (33), which is the firstorder perturbed Fock matrix in the $\mathrm{CO}$ basis. Whereas the diagonal blocks of $G^{\left(\mathcal{E}_{b}\right)}(\vec{k})$ determine $\epsilon^{\left(\mathcal{E}_{b}\right)}(\vec{k})$ the off-diagonal blocks determine $U^{\left(\mathcal{E}_{b}\right)}(\vec{k})$. In deriving (33) we have taken advantage of Eq. (28). Note, finally, that using (32) the first derivative of the density matrix Eq. (16) with respect to the field may be conveniently rewritten as

$$
D^{\left(\mathcal{E}_{a}\right)}(\vec{k})=C^{(0)}(\vec{k})\left[U^{\left(\mathcal{E}_{a}\right)}(\vec{k}) n+n U^{\left(\mathcal{E}_{a}\right) \dagger}(\vec{k})\right] C^{(0) \dagger}(\vec{k}) .
$$

\section{Second-order CPHF equations}

Let us start with the derivative of Eq. (24) with respect to a generic field direction $\mathcal{E}_{c}$ at zero field, which is given by

$$
\iota \Xi_{b}^{\left(\mathcal{E}_{c}\right)}(\vec{k})=\imath S(\vec{k}) C^{(0)}(\vec{k}) \frac{\partial U^{\left(\mathcal{E}_{c}\right)}(\vec{k})}{\partial k_{b}} C^{(0) \dagger}(\vec{k}) S(\vec{k})
$$

Since this result may not be completely evident a step-by step derivation is provided in the Appendix (see Eq. (A1)). In addition, differentiating Eq. (25) at zero field and using the antihermitian character of $\partial U^{\left(\mathcal{E}_{c}\right)}(\vec{k}) / \partial k_{b}$, yields

$$
\Omega_{b}^{\left(\mathcal{E}_{c}\right)}(\vec{k})=\imath \bar{\Xi}_{b}^{\left(\mathcal{E}_{c}\right)}(\vec{k})=\imath \Xi_{b}^{\left(\mathcal{E}_{c}\right)}(\vec{k})
$$

In order to obtain the second-order CPHF electric field equation for $C^{\left(\mathcal{E}_{b}, \mathcal{E}_{c}\right)}$ we take the second derivative of Eq. (12) with respect to the fields $\mathcal{E}_{b}, \mathcal{E}_{c}$ (evaluated at zero field) which 
leads to

$$
\begin{aligned}
& F^{\left(\mathcal{E}_{b}, \mathcal{E}_{c}\right)}(\vec{k}) C^{(0)}(\vec{k})+P_{b, c} F^{\left(\mathcal{E}_{c}\right)}(\vec{k}) C^{\left(\mathcal{E}_{b}\right)}(\vec{k})+F(\vec{k}) C^{\left(\mathcal{E}_{b}, \mathcal{E}_{c}\right)}(\vec{k}) \\
& =S(\vec{k})\left[C^{\left(\mathcal{E}_{b}, \mathcal{E}_{c}\right)}(\vec{k}) \epsilon^{(0)}(\vec{k})+P_{b, c} C^{\left(\mathcal{E}_{b}\right)}(\vec{k}) \epsilon^{\left(\mathcal{E}_{c}\right)}(\vec{k})\right. \\
& \left.\quad+C^{(0)}(\vec{k}) \epsilon^{\left(\mathcal{E}_{b}, \mathcal{E}_{c}\right)}(\vec{k})\right]
\end{aligned}
$$

for an arbitrary geometry. Equation (38) is solved by writing

$$
C^{\left(\mathcal{E}_{b}, \mathcal{E}_{c}\right)}(\vec{k})=C^{(0)}(\vec{k}) U^{\left(\mathcal{E}_{b}, \mathcal{E}_{c}\right)}(\vec{k})
$$

and, then, determining $U^{\left(\mathcal{E}_{b}, \mathcal{E}_{c}\right)}(\vec{k})$ self-consistently, so that the normalization condition is satisfied and the matrix of Lagrange multipliers

$$
\begin{aligned}
\epsilon^{\left(\mathcal{E}_{b}, \mathcal{E}_{c}\right)}(\vec{k})= & {\left[\epsilon^{(0)}(\vec{k}) U^{\left(\mathcal{E}_{b}, \mathcal{E}_{c}\right)}(\vec{k})-U^{\left(\mathcal{E}_{b}, \mathcal{E}_{c}\right)}(\vec{k}) \epsilon^{(0)}(\vec{k})\right] } \\
& +P_{b, c}\left(G^{\left(\mathcal{E}_{b}\right)}(\vec{k}) U^{\left(\mathcal{E}_{c}\right)}(\vec{k})-U^{\left(\mathcal{E}_{c}\right)}(\vec{k}) \epsilon^{\left(\mathcal{E}_{b}\right)}(\vec{k})\right) \\
& +G^{\left(\mathcal{E}_{b}, \mathcal{E}_{c}\right)}(\vec{k})
\end{aligned}
$$

is block-diagonal. The latter condition requires that

$$
\begin{aligned}
{\left[\epsilon^{(0)}(\vec{k}) U^{\left(\mathcal{E}_{b}, \mathcal{E}_{c}\right)}(\vec{k})-U^{\left(\mathcal{E}_{b}, \mathcal{E}_{c}\right)}(\vec{k}) \epsilon^{(0)}(\vec{k})\right]_{N D} } \\
\quad=P_{b, c} U^{\left(\mathcal{E}_{c}\right)}(\vec{k}) \epsilon^{\left(\mathcal{E}_{b}\right)}(\vec{k})-\left(G^{\left(\mathcal{E}_{b}\right)}(\vec{k}) U^{\left(\mathcal{E}_{c}\right)}(\vec{k})\right)_{N D} \\
\quad-G_{N D}^{\left(\mathcal{E}_{b}, \mathcal{E}_{c}\right)}(\vec{k})
\end{aligned}
$$

in which the subscript $N D$ indicates the non-diagonal blocks. Equations (40) and (41) are obtained by multiplying Eq. (38) on the left with $C^{(0) \dagger}(\vec{k})$.

As a consequence of (37), the explicit form of $G^{\left(\mathcal{E}_{b}, \mathcal{E}_{c}\right)}(\vec{k})$ is given by

$$
\begin{aligned}
G^{\left(\mathcal{E}_{b}, \mathcal{E}_{c}\right)}(\vec{k})= & C^{(0) \dagger}(\vec{k}) F^{\left(\mathcal{E}_{b}, \mathcal{E}_{c}\right)}(\vec{k}) C^{(0)}(\vec{k}) \\
= & C^{(0) \dagger}(\vec{k})\left[B^{\left(\mathcal{E}_{b}, \mathcal{E}_{c}\right)}(\vec{k})+T^{\left(\mathcal{E}_{b}, \mathcal{E}_{c}\right)}(\vec{k})\right. \\
& \left.+q^{\left(\mathcal{E}_{b}, \mathcal{E}_{c}\right)} S(\vec{k})\right] C^{(0)}(\vec{k})+P_{b, c} l \frac{\partial U^{\left(\mathcal{E}_{c}\right)}(\vec{k})}{\partial k_{b}} .
\end{aligned}
$$

Note that, in contrast to $U^{\left(\mathcal{E}_{b}\right)}(\vec{k})$, the diagonal blocks (occ-occ or virt-virt) of $U^{\left(\mathcal{E}_{b}, \mathcal{E}_{c}\right)}(\vec{k})$ are not zero, but are given by

$$
U_{D}^{\left(\mathcal{E}_{b}, \mathcal{E}_{c}\right)}(\vec{k})=-\frac{1}{2} P_{b, c}\left(U^{\left(\mathcal{E}_{b}\right) \dagger}(\vec{k}) U^{\left(\mathcal{E}_{c}\right)}(\vec{k})\right) .
$$

\section{Perturbation due to atomic displacements}

Equation (15) is valid at any geometry. Expanding about the equilibrium geometry the perturbed coefficients that are linear in the atomic displacements and zeroth-order in the field can be formally expressed as

$$
C^{\left(\mathcal{R}_{a}^{A}\right)}(\vec{k})=C^{(0)}(\vec{k}) U^{\left(\mathcal{R}_{a}^{A}\right)}(\vec{k}) .
$$

These coefficients will not appear in our final formula for the Raman intensities, but it is necessary to account for them in the intermediate steps of our derivation. Note that Eq. (44) is valid for all $k_{b}$.

The first derivative of the density matrix Eq. (8) with respect to the displacements may, then, be written as

$$
D^{\left(\mathcal{R}_{a}^{A}\right)}(\vec{k})=C^{\left(\mathcal{R}_{a}^{A}\right)}(\vec{k}) n C^{(0) \dagger}(\vec{k})+C^{(0)}(\vec{k}) n C^{\left(\mathcal{R}_{a}^{A}\right) \dagger}(\vec{k}),
$$

while the corresponding derivative of the normalization condition (Eq. (14)) is

$$
\begin{aligned}
& C^{\left(\mathcal{R}_{a}^{A}\right) \dagger}(\vec{k}) S(\vec{k}) C^{(0)}(\vec{k})+C^{(0) \dagger}(\vec{k}) S(\vec{k}) C^{\left(\mathcal{R}_{a}^{A}\right)}(\vec{k}) \\
& \quad=U^{\left(\mathcal{R}_{a}^{A}\right) \dagger}(\vec{k})+U^{\left(\mathcal{R}_{a}^{A}\right)}(\vec{k}) \\
& \quad=-C^{(0) \dagger}(\vec{k}) S^{\left(\mathcal{R}_{a}^{A}\right)}(\vec{k}) C^{(0)}(\vec{k}) .
\end{aligned}
$$

Moreover, at zero field, the derivative of the displacementperturbed coefficients with respect to the reciprocal space vector component $k_{b}$ is given by

$$
\begin{aligned}
\frac{\partial C^{\left(\mathcal{R}_{a}^{A}\right)}(\vec{k})}{\partial k_{b}} & =\frac{\partial C^{(0)}(\vec{k})}{\partial k_{b}} U^{\left(\mathcal{R}_{a}^{A}\right)}(\vec{k})+C^{(0)}(\vec{k}) \frac{\partial U^{\left(\mathcal{R}_{a}^{A}\right)}(\vec{k})}{\partial k_{b}} \\
& =C^{(0)}(\vec{k})\left[Q_{b}(\vec{k}) U^{\left(\mathcal{R}_{a}^{A}\right)}(\vec{k})+\frac{\partial U^{\left(\mathcal{R}_{a}^{A}\right)}(\vec{k})}{\partial k_{b}}\right] .
\end{aligned}
$$

\section{Second-order mixed derivative of the total energy with respect to $\mathcal{R}_{a}^{A}$ and $\mathcal{E}_{b}$.}

The mixed second derivative of the total energy with respect to the atomic displacement and the electric field (along $b$ )

$$
\begin{aligned}
\left.\frac{\partial}{\partial \mathcal{E}_{b}} \frac{\partial E^{T O T}}{\partial \mathcal{R}_{a}^{A}}\right|_{\mathcal{R}_{0}}= & \operatorname{Tr}\left(\frac{1}{2}\left(H^{\left(\mathcal{R}_{a}^{A}\right)}+\mathfrak{F}^{\left(\mathcal{R}_{a}^{A}\right)}\right) D^{\left(\mathcal{E}_{b}\right)}\right. \\
& +\frac{1}{2}\left[\Omega_{b}^{\left(\mathcal{R}_{a}^{A}\right)}+\left(\mathfrak{F}^{\left(\mathcal{R}_{a}^{A}\right)}\right)^{\left(\mathcal{E}_{b}\right)}\right] D \\
& \left.-S^{\left(\mathcal{R}_{a}^{A}\right)} D_{W}^{\left(\mathcal{E}_{b}\right)}\right)_{\mathcal{R}_{0}}-Z_{A} \delta_{a b},
\end{aligned}
$$

evaluated at zero field, was presented in our previous paper (Ref. 17). Now, however, we have changed the notation by introducing the (derivative of the) Fock matrix

$$
\begin{aligned}
\mathfrak{F}^{\left(\mathcal{R}_{a}^{A}\right)}= & h^{\left(\mathcal{R}_{a}^{A}\right)}+\left(\mathfrak{B}^{\left(\mathcal{R}_{a}^{A}\right)}+\mathfrak{T}^{\left(\mathcal{R}_{a}^{A}\right)}+\mathfrak{q}^{\left(\mathcal{R}_{a}^{A}\right)} S+q S^{\left(\mathcal{R}_{a}^{A}\right)}\right) \\
& +\sum_{b} \mathcal{E}_{b} \Omega_{b}^{\left(\mathcal{R}_{a}^{A}\right)}
\end{aligned}
$$

in which

$$
\mathfrak{B}_{\mu \nu \vec{g}}^{\left(\mathcal{R}_{a}^{A}\right)}=\sum_{\rho \tau \vec{h} \vec{n}} D_{\rho \overrightarrow{0} \tau \vec{n}} \frac{\partial}{\partial \mathcal{R}_{a}^{A}}\left(\mu^{\overrightarrow{0}} \nu^{\vec{g}} \| \rho^{\vec{h}} \tau^{\vec{h}+\vec{n}}\right)
$$

with an analogous expressions for $\mathfrak{T}^{\left(\mathcal{R}_{a}^{A}\right)}$

$$
\mathfrak{T}^{\left(\mathcal{R}_{a}^{A}\right)}=D \mathcal{T}^{\left(\mathcal{R}_{a}^{A}\right)}
$$

and $\mathfrak{q}^{\left(\mathcal{R}_{a}^{A}\right)}$. Once again, the derivatives with respect to nuclear displacements are to be obtained while holding the crystal orbital coefficients (and, hence, the density matrix) fixed at their equilibrium geometry values. This results from utilizing the eigenvalue-weighted density matrix, $D_{W}$, as is customary in quantum chemistry. ${ }^{46,47} D_{W}$ is defined in reciprocal space in a manner analogous to the ordinary density matrix (cf. Eq. (7)),

$$
D_{W}(\vec{k})=C(\vec{k}) \epsilon(\vec{k}) n C^{\dagger}(\vec{k}) .
$$


Its derivative with respect to the electric field component along direction $b$ may be obtained at zero field as

$$
\begin{aligned}
D_{W}^{\left(\mathcal{E}_{b}\right)}(\vec{k})= & C^{\left(\mathcal{E}_{b}\right)}(\vec{k}) \epsilon^{(0)}(\vec{k}) n C^{(0) \dagger}(\vec{k}) \\
& +C^{(0)}(\vec{k}) \epsilon^{\left(\mathcal{E}_{b}\right)}(\vec{k}) n C^{(0) \dagger}(\vec{k}) \\
& +C^{(0)}(\vec{k}) \epsilon^{(0)}(\vec{k}) n C^{\left(\mathcal{E}_{b}\right) \dagger}(\vec{k})
\end{aligned}
$$

The second derivatives of the eigenvalue-weighted density matrix with respect to electric field components $b$ and $c$, that will be needed in Sec. III, can be expressed (at zero field) as

$$
\begin{aligned}
D_{W}^{\left(\mathcal{E}_{b}, \mathcal{E}_{c}\right)}(\vec{k})= & \left(C^{\left(\mathcal{E}_{b}, \mathcal{E}_{c}\right)}(\vec{k}) \epsilon^{(0)}(\vec{k}) n C^{(0) \dagger}(\vec{k})\right. \\
& +P_{b, c}\left[C^{\left(\mathcal{E}_{b}\right)}(\vec{k}) \epsilon^{\left(\mathcal{E}_{c}\right)}(\vec{k}) n C^{(0) \dagger}(\vec{k})\right. \\
& \left.+C^{\left(\mathcal{E}_{b}\right)}(\vec{k}) \epsilon^{(0)}(\vec{k}) n C^{\left(\mathcal{E}_{c}\right) \dagger}(\vec{k})\right] \\
& +C^{(0)}(\vec{k}) \epsilon^{\left(\mathcal{E}_{b}, \mathcal{E}_{c}\right)}(\vec{k}) n C^{(0) \dagger}(\vec{k}) \\
& +P_{b, c}\left[C^{(0)}(\vec{k}) \epsilon^{\left(\mathcal{E}_{b}\right)}(\vec{k}) n C^{\left(\mathcal{E}_{c}\right) \dagger}(\vec{k})\right] \\
& \left.+C^{(0)}(\vec{k}) \epsilon^{(0)}(\vec{k}) n C^{\left(\mathcal{E}_{b}, \mathcal{E}_{c}\right) \dagger}(\vec{k})\right) .
\end{aligned}
$$

This result may be written in the more compact form

$$
D_{W}^{\left(\mathcal{E}_{b}, \mathcal{E}_{c}\right)}(\vec{k})=C^{(0)}(\vec{k})\left[A_{1}(\vec{k})+A_{2}(\vec{k})\right] C^{(0) \dagger}(\vec{k}),
$$

where

$$
\begin{aligned}
A_{1}(\vec{k})= & U^{\left(\mathcal{E}_{b}, \mathcal{E}_{c}\right)}(\vec{k}) \epsilon^{(0)}(\vec{k}) n \\
& +P_{b, c} U^{\left(\mathcal{E}_{b}\right)}(\vec{k}) \epsilon^{(0)}(\vec{k}) n U^{\left(\mathcal{E}_{c}\right) \dagger}(\vec{k}) \\
& +\epsilon^{(0)}(\vec{k}) n U^{\left(\mathcal{E}_{b}, \mathcal{E}_{c}\right) \dagger}(\vec{k}), \\
A_{2}(\vec{k})= & P_{b, c}\left(U^{\left(\mathcal{E}_{b}\right)}(\vec{k}) \epsilon^{\left(\mathcal{E}_{c}\right)}(\vec{k}) n+\epsilon^{\left(\mathcal{E}_{b}\right)}(\vec{k}) n U^{\left(\mathcal{E}_{c}\right) \dagger}(\vec{k})\right) \\
& +\epsilon^{\left(\mathcal{E}_{b}, \mathcal{E}_{c}\right)}(\vec{k}) n \\
= & P_{b, c}\left(\epsilon^{\left(\mathcal{E}_{b}\right)}(\vec{k}) n U^{\left(\mathcal{E}_{c}\right) \dagger}(\vec{k})+G^{\left(\mathcal{E}_{b}\right)}(\vec{k}) U^{\left(\mathcal{E}_{c}\right)}(\vec{k}) n\right) \\
& +\left(\epsilon^{(0)}(\vec{k}) U^{\left(\mathcal{E}_{b}, \mathcal{E}_{c}\right)}(\vec{k})-U^{\left(\mathcal{E}_{b}, \mathcal{E}_{c}\right)}(\vec{k}) \epsilon^{(0)}(\vec{k})\right. \\
& \left.+G^{\left(\mathcal{E}_{b}, \mathcal{E}_{c}\right)}(\vec{k})\right) n .
\end{aligned}
$$

\section{RAMAN INTENSITIES}

Our goal is to obtain a computable expression for the Raman tensor elements. The first step is to differentiate Eq. (48) with respect to a generic field direction $\mathcal{E}_{c}$ prior to evaluating this expression at zero field:

$$
\begin{aligned}
\left.\frac{\partial^{2}}{\partial \mathcal{E}_{b} \partial \mathcal{E}_{c}} \frac{\partial E^{T O T}}{\partial \mathcal{R}_{a}^{A}}\right|_{\mathcal{E}=0, \mathcal{R}_{0}} \\
=\operatorname{Tr}\left\{\frac{1}{2}\left(H^{\left(\mathcal{R}_{a}^{A}\right)}+\mathfrak{F}^{\left(\mathcal{R}_{a}^{A}\right)}\right)^{(0)} D^{\left(\mathcal{E}_{b}, \mathcal{E}_{c}\right)}\right. \\
+\frac{1}{2} P_{b, c}\left[\Omega_{b}^{\left(\mathcal{R}_{a}^{A}\right)}+\left(\mathfrak{F}^{\left(\mathcal{R}_{a}^{A}\right)}\right)^{\left(\mathcal{E}_{b}\right)}\right] D^{\left(\mathcal{E}_{c}\right)} \\
+\frac{1}{2}\left[P_{b, c} \bar{\Xi}_{b}^{\left(\mathcal{R}_{a}^{A}, \mathcal{E}_{c}\right)}+\left(\mathfrak{F}^{\left(\mathcal{R}_{a}^{A}\right)}\right)^{\left(\mathcal{E}_{b}, \mathcal{E}_{c}\right)}\right] D^{(0)} \\
\left.\quad-S^{\left(\mathcal{R}_{a}^{A}\right)} D_{W}^{\left(\mathcal{E}_{b}, \mathcal{E}_{c}\right)}\right\}_{\mathcal{R}_{0}, \mathcal{E}=0} \cdot
\end{aligned}
$$

Next we discuss the various features of this equation and, then, perform some manipulations leading to a formula that is simpler and more suitable for implementation.

\section{A. The mixed derivative of the electron-electron and spheropole terms}

The $\mathfrak{B}^{\left(\mathcal{R}_{a}^{A}\right)}, \mathfrak{T}^{\left(\mathcal{R}_{a}^{A}\right)}$, and $\mathfrak{q}^{\left(\mathcal{R}_{a}^{A}\right)}$ quantities, defined in Eqs. (49)-(51), contain the field-dependent density matrix, and gradient of a field-free integral. The first and second derivative of these terms with respect to the electric field components along $b$ and $c$ are, then, easily obtained by substituting the field-perturbed density matrix in the relevant expressions.

\section{B. Gradients of the $\Omega$ and $\Omega^{\left(\mathcal{E}_{c}\right)}$ matrices}

The $\Omega_{b}$ term in Eq. (49) is linear (and higher order) in the field; it vanishes for $\mathcal{E}=0$. Hence, for the Raman effect we are interested in the first derivative of the terms involving this quantity in Eq. (48), i.e.,

$$
P_{b, c} \sum_{\vec{k}} \operatorname{Tr}\left(\Omega_{b}^{\left(\mathcal{R}_{a}^{A}\right)}(\vec{k}) D^{\left(\mathcal{E}_{c}\right)}(\vec{k})+\imath \bar{\Xi}_{b}^{\left(\mathcal{R}_{a}^{A}, \mathcal{E}_{c}\right)}(\vec{k}) D^{(0)}(\vec{k})\right) .
$$

The treatment of the $M_{b}$ part of $\Omega_{b}$ is trivial since $M_{b}$ is fieldindependent. So our focus is on obtaining a suitable expression for

$$
P_{b, c} \sum_{\vec{k}} \operatorname{Tr}\left(\iota \bar{\Xi}_{b}^{\left(\mathcal{R}_{a}^{A}\right)}(\vec{k}) D^{\left(\mathcal{E}_{c}\right)}(\vec{k})+\imath \bar{\Xi}_{b}^{\left(\mathcal{R}_{a}^{A}, \mathcal{E}_{c}\right)}(\vec{k}) D^{(0)}(\vec{k})\right) .
$$

In order to simplify the treatment the non-hermitized form of $\Xi_{b}(\vec{k})$ is used below, but it is straightforward to convert to the hermitian conjugate.

To obtain the first term of Eq. (60), an explicit expression for $\Xi_{b}^{\left(\mathcal{R}_{a}^{A}\right)}(\vec{k})$ is needed. To that end we differentiate Eq. (24) with respect to $\mathcal{R}_{a}^{A}$ at zero-field to obtain

$$
\begin{aligned}
\Xi_{b}^{\left(\mathcal{R}_{a}^{A}\right)}(\vec{k})= & S^{\left(\mathcal{R}_{a}^{A}\right)}(\vec{k}) C^{(0)}(\vec{k}) Q_{b}(\vec{k}) C^{(0) \dagger}(\vec{k}) S(\vec{k})+S(\vec{k}) C^{(0)}(\vec{k}) Q_{b}(\vec{k}) C^{(0) \dagger}(\vec{k}) S^{\left(\mathcal{R}_{a}^{A}\right)}(\vec{k}) \\
& +S(\vec{k}) C^{(0)}(\vec{k})\left[\frac{\partial U^{\left(\mathcal{R}_{a}^{A}\right)}(\vec{k})}{\partial k_{b}}-Q_{b}(\vec{k}) C^{(0) \dagger}(\vec{k}) S^{\left(\mathcal{R}_{a}^{A}\right)}(\vec{k}) C^{(0)}(\vec{k})\right] C^{(0) \dagger}(\vec{k}) S(\vec{k})
\end{aligned}
$$


This result may not be immediately evident. However, a complete derivation is presented in the Appendix (see Eq. (A2)). Using the result of Eqs. (16) and (61) for $D^{\left(\mathcal{E}_{c}\right)}$ we find that

$$
\begin{aligned}
& \sum_{\vec{k}} \operatorname{Tr}\left(\imath \Xi_{b}^{\left(\mathcal{R}_{a}^{A}\right)}(\vec{k}) D^{\left(\mathcal{E}_{c}\right)}(\vec{k})\right) \\
& \quad=\imath \sum_{\vec{k}} \operatorname{Tr}\left(\left[C^{(0) \dagger}(\vec{k}) S^{\left(\mathcal{R}_{a}^{A}\right)}(\vec{k}) C^{(0)}(\vec{k}) Q_{b}(\vec{k})+\frac{\partial U^{\left(\mathcal{R}_{a}^{A}\right)}(\vec{k})}{\partial k_{b}}\right]\left[U^{\left(\mathcal{E}_{c}\right)}(\vec{k}) n-n U^{\left(\mathcal{E}_{c}\right)}(\vec{k})\right]\right) .
\end{aligned}
$$

In order to eliminate $\partial U^{\left(\mathcal{R}_{a}^{A}\right)}(\vec{k}) / \partial k_{b}$ from the last term in Eq. (62) we consider the integral

$$
\sum_{\vec{k}}^{B Z} \frac{\partial}{\partial k_{b}}\left[U^{\left(\mathcal{R}_{a}^{A}\right)}(\vec{k}) U^{\left(\mathcal{E}_{c}\right)}(\vec{k})\right]=0
$$

which is zero since we may assume ${ }^{48}$ that both $U^{\left(\mathcal{R}_{a}^{A}\right)}(\vec{k})$ and $U^{\left(\mathcal{E}_{c}\right)}(\vec{k})$ are the same at opposite boundaries of the first
Brillouin zone, i.e., at $\pm \vec{k}$. It, then, follows that

$$
\sum_{\vec{k}}^{B Z} \frac{\partial U^{\left(\mathcal{R}_{a}^{A}\right)}(\vec{k})}{\partial k_{b}} U^{\left(\mathcal{E}_{c}\right)}(\vec{k})=-\sum_{\vec{k}}^{B Z} U^{\left(\mathcal{R}_{a}^{A}\right)}(\vec{k}) \frac{\partial U^{\left(\mathcal{E}_{c}\right)}(\vec{k})}{\partial k_{b}}
$$

and, by the same token,

$$
\sum_{\vec{k}}^{B Z} \frac{\partial U^{\left(\mathcal{E}_{c}\right)}(\vec{k})}{\partial k_{b}} U^{\left(\mathcal{R}_{a}^{A}\right)}(\vec{k})=-\sum_{\vec{k}}^{B Z} U^{\left(\mathcal{E}_{c}\right)}(\vec{k}) \frac{\partial U^{\left(\mathcal{R}_{a}^{A}\right)}(\vec{k})}{\partial k_{b}} .
$$

Substitution of Eqs. (64) and (65) into Eq. (62) gives

$$
\begin{aligned}
\sum_{\vec{k}} \operatorname{Tr}\left(\imath \Xi_{b}^{\left(\mathcal{R}_{a}^{A}\right)}(\vec{k}) D^{\left(\mathcal{E}_{c}\right)}(\vec{k})\right)= & \iota \sum_{\vec{k}} \operatorname{Tr}\left(C^{(0) \dagger}(\vec{k}) S^{\left(\mathcal{R}_{a}^{A}\right)}(\vec{k}) C^{(0)}(\vec{k}) Q_{b}(\vec{k})\left[U^{\left(\mathcal{E}_{c}\right)}(\vec{k}) n-n U^{\left(\mathcal{E}_{c}\right)}(\vec{k})\right]\right. \\
& \left.-U^{\left(\mathcal{R}_{a}^{A}\right)}(\vec{k})\left[\frac{\partial U^{\left(\mathcal{E}_{c}\right)}(\vec{k})}{\partial k_{b}} n-n \frac{\partial U^{\left(\mathcal{E}_{c}\right)}(\vec{k})}{\partial k_{b}}\right]\right)
\end{aligned}
$$

as desired. We now turn to the second contribution of Eq. (60). An expression for $\Xi_{b}^{\left(\mathcal{R}_{a}^{A}, \mathcal{E}_{c}\right)}(\vec{k})$ may be found by differentiating Eq. (37) with respect to displacements (note that all quantities in that equation, including $C^{(0)}$, depend upon $\mathcal{R}_{a}^{A}$ ). This yields

$$
\begin{aligned}
\Xi_{b}^{\left(\mathcal{R}_{a}^{A}, \mathcal{E}_{c}\right)}(\vec{k})= & S^{\left(\mathcal{R}_{a}^{A}\right)}(\vec{k}) C^{(0)}(\vec{k}) \frac{\partial U^{\left(\mathcal{E}_{c}\right)}(\vec{k})}{\partial k_{b}} C^{(0) \dagger}(\vec{k}) S(\vec{k})+S(\vec{k}) C^{(0)}(\vec{k}) \frac{\partial U^{\left(\mathcal{E}_{c}\right)}(\vec{k})}{\partial k_{b}} C^{(0) \dagger}(\vec{k}) S^{\left(\mathcal{R}_{a}^{A}\right)}(\vec{k}) \\
& +S(\vec{k}) C^{(0)}(\vec{k})\left[U^{\left(\mathcal{R}_{a}^{A}\right)}(\vec{k}) \frac{\partial U^{\left(\mathcal{E}_{c}\right)}(\vec{k})}{\partial k_{b}}+\left(\frac{\partial U^{\left(\mathcal{E}_{c}\right)}(\vec{k})}{\partial k_{b}}\right)^{\left(\mathcal{R}_{a}^{A}\right)}\right. \\
& \left.+\frac{\partial U^{\left(\mathcal{E}_{c}\right)}(\vec{k})}{\partial k_{b}} U^{\left(\mathcal{R}_{a}^{A}\right) \dagger}(\vec{k})\right] C^{(0) \dagger}(\vec{k}) S(\vec{k}),
\end{aligned}
$$

which leads to

$$
\begin{aligned}
& \sum_{\vec{k}} \operatorname{Tr}\left(\imath \Xi_{b}^{\left(\mathcal{R}_{a}^{A}, \mathcal{E}_{c}\right)}(\vec{k}) D^{(0)}(\vec{k})\right) \\
& \quad=\imath \sum_{\vec{k}} \operatorname{Tr}\left(U^{\left(\mathcal{R}_{a}^{A}\right)}(\vec{k})\left[\frac{\partial U^{\left(\mathcal{E}_{c}\right)}(\vec{k})}{\partial k_{b}} n-n \frac{\partial U^{\left(\mathcal{E}_{c}\right)}(\vec{k})}{\partial k_{b}}\right]+C^{(0) \dagger}(\vec{k}) S^{\left(\mathcal{R}_{a}^{A}\right)}(\vec{k}) C^{(0)}(\vec{k}) \frac{\partial U^{\left(\mathcal{E}_{c}\right)}(\vec{k})}{\partial k_{b}} n\right) .
\end{aligned}
$$

Once again, for convenience, the individual steps leading to Eq. (68) have been relegated to the Appendix (see Eq. (A3)). Note that Eq. (46) has been used here. By combining Eqs. (66) and (68), reintroducing the $M_{b}$ term, and recovering the hermitized 
form for $Q_{b}$ we, finally, obtain

$$
\begin{aligned}
P_{b, c} & \sum_{\vec{k}} \operatorname{Tr}\left\{\Omega_{b}^{\left(\mathcal{R}_{a}^{A}\right)}(\vec{k}) D^{\left(\mathcal{E}_{c}\right)}(\vec{k})+\Omega_{b}^{\left(\mathcal{R}_{a}^{A}, \mathcal{E}_{c}\right)}(\vec{k}) D^{(0)}(\vec{k})\right\} \\
= & P_{b, c} \sum_{\vec{k}} \operatorname{Tr}\left\{\bar{M}_{b}^{\left(\mathcal{R}_{a}^{A}\right)}(\vec{k}) D^{\left(\mathcal{E}_{c}\right)}(\vec{k})\right. \\
& \left.+\imath C^{(0) \dagger}(\vec{k}) S^{\left(\mathcal{R}_{a}^{A}\right)}(\vec{k}) C^{(0)}(\vec{k})\left(\bar{Q}_{b}(\vec{k})\left[U^{\left(\mathcal{E}_{c}\right)}(\vec{k}) n-n U^{\left(\mathcal{E}_{c}\right)}(\vec{k})\right]+\frac{\partial U^{\left(\mathcal{E}_{c}\right)}(\vec{k})}{\partial k_{b}} n\right)\right\} .
\end{aligned}
$$

This expression has the key feature that it does not contain $U^{\left(\mathcal{R}_{a}^{A}\right)}(\vec{k})$ and, thus, does not require solution of the set of coupledperturbed equations with respect to atomic displacements. Moreover, we will see that it cancels in large part with terms contained inside the $D_{W}$ term of Eq. (58), as discussed in Subsection III C.

\section{A compact, computable formula for Raman intensities}

It is convenient at this point to pick out those terms in Eq. (57) that involve either $Q_{b}(\vec{k})$ or $\partial U^{\left(\mathcal{E}_{c}\right)}(\vec{k}) / \partial k_{b}$. These terms arise from $\epsilon^{\left(\mathcal{E}_{b}\right)}(\vec{k}), G^{\left(\mathcal{E}_{b}\right)}(\vec{k})$, and $G^{\left(\mathcal{E}_{b}, \mathcal{E}_{c}\right)}(\vec{k})$ (see Eqs. (33) ff.) and (42). After substituting into Eq. (55) we find that their contribution to the $\operatorname{Tr}\left(S^{\left(\mathcal{R}_{a}^{A}\right)} D_{W}^{\left(\mathcal{E}_{b}, \mathcal{E}_{c}\right)}\right)$ term in Eq. (58) is

$$
-P_{b, c} \sum_{\vec{k}} \operatorname{Tr}\left\{l C^{(0) \dagger}(\vec{k}) S^{\left(\mathcal{R}_{a}^{A}\right)}(\vec{k}) C^{(0)}(\vec{k})\left(-\mathcal{P}_{o c c} \bar{Q}_{b}(\vec{k}) n U^{\left(\mathcal{E}_{c}\right)}(\vec{k})+\bar{Q}_{b}(\vec{k}) U^{\left(\mathcal{E}_{c}\right)}(\vec{k}) n+\frac{\partial U^{\left(\mathcal{E}_{c}\right)}(\vec{k})}{\partial k_{b}} n\right)\right\}
$$

where $\mathcal{P}_{o c c}$ is the projector for the occupied space. The latter accounts for the fact that $\epsilon^{\left(\mathcal{E}_{b}\right)}(\vec{k})$ is block-diagonal. These terms occur with a negative sign and, therefore, exactly cancel with the corresponding terms in Eq. (69) except for the projector. Due to the projector there is a term left over when the two are combined, namely,

$$
P_{b, c} \sum_{k} \operatorname{Tr}\left\{l C^{(0) \dagger}(\vec{k}) S^{\left(\mathcal{R}_{a}^{A}\right)}(\vec{k}) C^{(0)}(\vec{k})\left(\mathcal{P}_{\text {virt }} \bar{Q}_{b}(\vec{k}) n U^{\left(\mathcal{E}_{c}\right)}(\vec{k})\right)\right\}
$$

with $\mathcal{P}_{\text {virt }}=I-\mathcal{P}_{\text {occ }}$. We define a modified form of $D_{W}^{\left(\mathcal{E}_{b}, \mathcal{E}_{c}\right)}(\vec{k})$, i.e.,

$$
D_{W}^{\prime\left(\mathcal{E}_{b}, \mathcal{E}_{c}\right)}(\vec{k})=C^{(0)}(\vec{k})\left[A_{1}(\vec{k})+A_{2}^{\prime}(\vec{k})-P_{b, c}\left(l \mathcal{P}_{v i r t} \bar{Q}_{b}(\vec{k}) n U^{\left(\mathcal{E}_{c}\right)}(\vec{k})\right)\right] C^{(0) \dagger}(\vec{k})
$$

where the appropriate $\mathrm{Q}_{b}$ term has been added and $A_{2}(\vec{k})$ has been replaced by $A_{2}^{\prime}(\vec{k})$,

$$
\begin{aligned}
A_{2}^{\prime}(\vec{k})= & P_{b, c}\left[X^{\left(\mathcal{E}_{b}\right)}(\vec{k}) U^{\left(\mathcal{E}_{c}\right)}(\vec{k}) n\right. \\
& \left.-\mathcal{P}_{o c c} X^{\left(\mathcal{E}_{b}\right)}(\vec{k}) n U^{\left(\mathcal{E}_{c}\right)}(\vec{k})\right]+X^{\left(\mathcal{E}_{b}, \mathcal{E}_{c}\right)}(\vec{k}) n \\
& +\left(\epsilon^{(0)}(\vec{k}) U^{\left(\mathcal{E}_{b}, \mathcal{E}_{c}\right)}(\vec{k})-U^{\left(\mathcal{E}_{b}, \mathcal{E}_{c}\right)}(\vec{k}) \epsilon^{(0)}(\vec{k})\right) n
\end{aligned}
$$

with

$$
\begin{aligned}
X^{\left(\mathcal{E}_{b}\right)}(\vec{k})= & C^{(0) \dagger}(\vec{k})\left[B^{\left(\mathcal{E}_{b}\right)}(\vec{k})+T^{\left(\mathcal{E}_{b}\right)}(\vec{k})+q^{\left(\mathcal{E}_{b}\right)} S(\vec{k})\right. \\
& \left.+\bar{M}_{b}(\vec{k})\right] C^{(0)}(\vec{k}) .
\end{aligned}
$$

Here $X^{\left(\mathcal{E}_{b}, \mathcal{E}_{c}\right)}(\vec{k})$ is a modified second-order $G^{\left(\mathcal{E}_{b}, \mathcal{E}_{c}\right)}(\vec{k})$ matrix after elimination of $\partial U^{\left(\mathcal{E}_{c}\right)}(\vec{k}) / \partial k_{b}$ as just described

$$
X^{\left(\mathcal{E}_{b}, \mathcal{E}_{c}\right)}(\vec{k})=C^{(0) \dagger}(\vec{k})\left[B^{\left(\mathcal{E}_{b}, \mathcal{E}_{c}\right)}(\vec{k})+T^{\left(\mathcal{E}_{b}, \mathcal{E}_{c}\right)}(\vec{k})+q^{\left(\mathcal{E}_{b}, \mathcal{E}_{c}\right)} S(\vec{k})\right] C^{(0)}(\vec{k}) .
$$

In order to account for the fact that the $\bar{\Xi}_{b}$ term in $\Omega_{b}$ is treated separately above from the $\bar{M}_{b}$ bar term it is convenient to define the modified Fock matrix derivative

$$
\begin{aligned}
\mathfrak{F}^{\left(\mathcal{R}_{a}^{A}\right)}= & \mathfrak{B}^{\left(\mathcal{R}_{a}^{A}\right)}+\mathfrak{T}^{\left(\mathcal{R}_{a}^{A}\right)}+\mathfrak{q}^{\left(\mathcal{R}_{a}^{A}\right)} S+q S^{\left(\mathcal{R}_{a}^{A}\right)} \\
& +\sum_{b} \mathcal{E}_{b} \bar{M}_{b}^{\left(\mathcal{R}_{a}^{A}\right)} .
\end{aligned}
$$

Note that $\mathfrak{F}^{\left(\mathcal{R}_{a}^{A}\right)}$ contains terms where the field(s) appear only either in the density matrix as a multiplicative factor or not at all. Then, finally, inserting (72) in (58), we obtain a compact 
computable expression for the Raman tensor elements

$$
\begin{aligned}
\left.\frac{\partial^{2}}{\partial \mathcal{E}_{b} \partial \mathcal{E}_{c}} \frac{\partial E^{T O T}}{\partial \mathcal{R}_{a}^{A}}\right|_{\mathcal{E}=0, \mathcal{R}_{0}} \\
=\operatorname{Tr}\left\{\frac{1}{2}\left(H^{\left(\mathcal{R}_{a}^{A}\right)}+\mathfrak{F}^{\prime\left(\mathcal{R}_{a}^{A}\right)}\right)^{(0)} D^{\left(\mathcal{E}_{b}, \mathcal{E}_{c}\right)}\right. \\
\quad+\frac{1}{2} P_{b, c}\left[\left(\bar{M}_{b}^{\left(\mathcal{R}_{a}^{A}\right)}+\left(\mathfrak{F}^{\prime\left(\mathcal{R}_{a}^{A}\right)}\right)^{\left(\mathcal{E}_{b}\right)}\right) D^{\left(\mathcal{E}_{c}\right)}\right] \\
\left.\quad+\frac{1}{2}\left(\mathfrak{F}^{\prime\left(\mathcal{R}_{a}^{A}\right)}\right)^{\left(\mathcal{E}_{b}, \mathcal{E}_{c}\right)} D^{(0)}-S^{\left(\mathcal{R}_{a}^{A}\right)} D_{W}^{\prime\left(\mathcal{E}_{b}, \mathcal{E}_{c}\right)}\right\}_{\mathcal{R}_{0}}
\end{aligned}
$$

\section{Remarks}

Taking into account the statement immediately below Eq. (76), it is clear that there are no entities in our final formula that must be obtained by double differentiation with respect to the field and with respect to atomic positions. Most importantly, no perturbation equations for the atomic displacements need be solved. All quantities involved are obtained either through a CPHF calculation or by determining the gradient of an integral as in a standard analytical geometry optimization. Thus, the computational cost scales favorably with the size of the unit cell and is, in fact, a fraction of the cost of obtaining the fundamental frequencies, if symmetry is not present. ${ }^{49}$ Equation (77) is a fully analytical expression in direct space and AO basis for obtaining the Raman intensities in periodic systems. This is the expression that has been implemented in the CRYSTAL code. ${ }^{34}$

\section{E. Extension to CPKS version of DFT}

Let us now consider the extension of Eq. (77) to the CPKS version of DFT. We derive the expression for a hybrid functional, such as B3LYP, since other approximations, like the local density approximation (LDA) or generalized gradient approximations (GGA) can be considered as special cases. Meta-GGA functionals can be treated by an extension of the method we present.

In DFT calculations, the total electronic energy is separated into parts ${ }^{50}$

$$
E_{D F T}^{T O T}\left(a_{X}\right)=E^{T}+E^{V}+E^{J}+a_{X} E_{H F}^{X}+E^{\Omega}+E_{R}^{X C}\left(a_{X}\right) .
$$

In Eq. (78) $E^{T}, E^{V}$, and $E^{J}$ are the kinetic, electron-nuclear, and Coulomb interaction energies, respectively, and they are the same as in the HF treatment. $E^{\Omega}$, which is also the same as in $\mathrm{HF}$, represents the contribution due to the electric field while $E_{H F}^{X}$ is the HF exchange contribution

$$
\begin{gathered}
E_{H F}^{X}=\operatorname{Tr}\left(B^{X} D\right), \\
B_{\mu, \nu \vec{g}}^{X}=-\frac{1}{2} \sum_{\rho, \tau, h, n} D_{\rho \tau \vec{n}}\left(\mu \rho^{\vec{h}} \mid \nu^{\vec{g}} \tau^{\vec{h}+\vec{n}}\right),
\end{gathered}
$$

which is multiplied by the scaling factor $a_{X}$ to give the fraction of "exact" exchange in the functional. Finally, $E_{R}^{X C}\left(a_{X}\right)$ is the remaining exchange-correlation part of the electron-electron repulsion energy.

For simplicity, we consider here again the case of a closed shell system, so that $E_{R}^{X C}$ may be written as the numerical integral (we drop the explicit dependence of $E_{R}^{X C}$ on $a_{X}$ for notational convenience)

$$
E_{R}^{X C}=\sum_{i} w_{i} f^{X C}\left(\rho,|\nabla \rho|^{2}\right)_{\mathbf{r}_{i}} .
$$

The value of $f^{X C}$ is calculated at points $\mathbf{r}_{i}$ on a grid and multiplied by the Becke weight ${ }^{51,52} w_{i}$ associated with each point, according to an atomic partition. The density and its electronic gradient are obtained from

$$
\begin{gathered}
\rho\left(\mathbf{r}_{i}\right)=\sum_{\mu \nu \vec{g}} D_{\mu \nu \vec{g}} \phi_{\mu}^{\overrightarrow{0}}\left(\mathbf{r}_{i}\right) \phi_{\nu}^{\vec{g}}\left(\mathbf{r}_{i}\right), \\
\nabla \rho\left(\mathbf{r}_{i}\right)=\sum_{\mu \nu \vec{g}} D_{\mu \nu \vec{g}}\left[\nabla\left(\phi_{\mu}^{\overrightarrow{0}} \phi_{\nu}^{\vec{g}}\right)\right]_{\mathbf{r}_{i}},
\end{gathered}
$$

where $\phi_{\mu}$ and $\phi_{v}$ are basis functions. Except for $E_{R}^{X C}$ (and the scaling factor $a_{X}$ ), Eq. (78) is identical to (6). Therefore, Eq. (77) is obtained following the same route as for HartreeFock, and to that we add the derivative of $E_{R}^{X C}$ with respect to the displacement and two fields. Note that in the DFT case the exchange part of $\mathfrak{B}^{\left(\mathcal{R}_{a}^{A}\right)}$ in (77) will be scaled by $a_{X}$, and that $D_{W}^{\prime}{ }^{\left(\mathcal{E}_{b}\right)}$ will contain DFT eigenvalues. Differentiation of Eqs. (82) and (83) with respect to nuclear displacements, while holding the crystal orbital coefficients fixed (as in Eq. (48)), gives

$$
\begin{array}{r}
\rho^{\left(\mathcal{R}_{a}^{A}\right)}\left(\mathbf{r}_{i}\right)=\sum_{\mu \nu \vec{g}} D_{\mu \nu \vec{g}}\left[\frac{\partial}{\partial \mathcal{R}_{a}^{A}}\left(\phi_{\mu}^{\overrightarrow{0}} \phi_{\nu}^{\vec{g}}\right)\right]_{\mathbf{r}_{i}}, \\
\nabla \rho^{\left(\mathcal{R}_{a}^{A}\right)}\left(\mathbf{r}_{i}\right)=\sum_{\mu \nu \vec{g}} D_{\mu \nu \vec{g}}\left[\frac{\partial}{\partial \mathcal{R}_{a}^{A}} \nabla\left(\phi_{\mu}^{\overrightarrow{0}} \phi_{\nu}^{\vec{g}}\right)\right]_{\mathbf{r}_{i}} .
\end{array}
$$

For the derivative of $\mathrm{E}_{R}^{X C}$ with respect to the displacement and two fields we can use as a starting point the closed shell expression for the $E_{R}^{X C}$ contribution to the Born charges tensor obtained in Ref. 17,

$$
\begin{aligned}
\left.\frac{\partial E_{R}^{X C\left(\mathcal{R}_{a}^{A}\right)}}{\partial \mathcal{E}_{b}}\right|_{\mathcal{R}_{0}} & \sum_{i} w_{i}\left\{\left[\frac{\partial^{2} f^{X C}}{\partial \rho^{2}} \frac{\partial \rho}{\partial \mathcal{E}_{b}}+\frac{\partial^{2} f^{X C}}{\partial \rho \partial|\nabla \rho|^{2}} \frac{\partial|\nabla \rho|^{2}}{\partial \mathcal{E}_{b}}\right] \rho^{\left(\mathcal{R}_{a}^{A}\right)}\right. \\
& +2\left[\frac{\partial^{2} f^{X C}}{\partial\left(|\nabla \rho|^{2}\right)^{2}} \frac{\partial|\nabla \rho|^{2}}{\partial \mathcal{E}_{b}} \nabla \rho+\frac{\partial^{2} f^{X C}}{\partial \rho \partial|\nabla \rho|^{2}} \frac{\partial \rho}{\partial \mathcal{E}_{b}} \nabla \rho\right. \\
& \left.+\frac{\partial f^{X C}}{\partial|\nabla \rho|^{2}} \frac{\partial \nabla \rho}{\partial \mathcal{E}_{b}}\right] \cdot \nabla \rho^{\left(\mathcal{R}_{a}^{A}\right)} \\
& \left.+\frac{\partial f^{X C}}{\partial \rho} \frac{\partial \rho^{\left(\mathcal{R}_{a}^{A}\right)}}{\partial \mathcal{E}_{b}}+2 \frac{\partial f^{X C}}{\partial|\nabla \rho|^{2}} \nabla \rho \cdot \frac{\partial \nabla \rho^{\left(\mathcal{R}_{a}^{A}\right)}}{\partial \mathcal{E}_{b}}\right\}_{\mathbf{r}_{i}} \\
& +\sum_{i} \frac{\partial w_{i}}{\partial \mathcal{R}_{a}^{A}}\left\{\frac{\partial f^{X C}}{\partial \rho} \frac{\partial \rho}{\partial \mathcal{E}_{b}}+\frac{\partial f^{X C}}{\partial|\nabla \rho|^{2}} \frac{\partial|\nabla \rho|^{2}}{\partial \mathcal{E}_{b}}\right\}_{\mathbf{r}_{i}}
\end{aligned}
$$


Equation (86) was determined by taking advantage of the equality $\left[|\nabla \rho|^{2}\right]^{\left(\mathcal{R}_{a}^{A}\right)}=2 \nabla \rho \cdot \nabla \rho^{\left(\mathcal{R}_{a}^{A}\right)}$. Finally, to obtain the corresponding contribution to the Raman tensor we differentiate the above relation with respect to the electric field $\mathcal{E}_{c}$, which yields

$$
\begin{aligned}
& \left.\frac{\partial^{2} E_{R}^{X C\left(\mathcal{R}_{a}^{A}\right)}}{\partial \mathcal{E}_{b} \partial \mathcal{E}_{c}}\right|_{\mathcal{E}=0, \mathcal{R}_{0}}=\sum_{i} w_{i}\left\{\left[\frac{\partial^{3} f^{X C}}{\partial \rho^{3}} \frac{\partial \rho}{\partial \mathcal{E}_{b}} \frac{\partial \rho}{\partial \mathcal{E}_{c}}+\frac{\partial^{2} f^{X C}}{\partial \rho^{2}} \frac{\partial^{2} \rho}{\partial \mathcal{E}_{b} \partial \mathcal{E}_{c}}+P_{b, c} \frac{\partial^{3} f^{X C}}{\partial \rho^{2} \partial|\nabla \rho|^{2}} \frac{\partial \rho}{\partial \mathcal{E}_{b}} \frac{\partial|\nabla \rho|^{2}}{\partial \mathcal{E}_{c}}\right.\right. \\
& \left.+\frac{\partial^{3} f^{X C}}{\partial \rho\left(\partial|\nabla \rho|^{2}\right)^{2}} \frac{\partial|\nabla \rho|^{2}}{\partial \mathcal{E}_{b}} \frac{\partial|\nabla \rho|^{2}}{\partial \mathcal{E}_{c}}+\frac{\partial^{2} f^{X C}}{\partial \rho \partial|\nabla \rho|^{2}} \frac{\partial^{2}|\nabla \rho|^{2}}{\partial \mathcal{E}_{b} \partial \mathcal{E}_{c}}\right] \rho^{\left(\mathcal{R}_{a}^{A}\right)} \\
& +2\left[\left(\frac{\partial^{3} f^{X C}}{\partial\left(|\nabla \rho|^{2}\right)^{3}} \frac{\partial|\nabla \rho|^{2}}{\partial \mathcal{E}_{b}} \frac{\partial|\nabla \rho|^{2}}{\partial \mathcal{E}_{c}}+\frac{\partial^{2} f^{X C}}{\partial\left(|\nabla \rho|^{2}\right)^{2}} \frac{\partial^{2}|\nabla \rho|^{2}}{\partial \mathcal{E}_{b} \partial \mathcal{E}_{c}}+P_{b, c} \frac{\partial^{3} f^{X C}}{\partial\left(|\nabla \rho|^{2}\right)^{2} \partial \rho} \frac{\partial|\nabla \rho|^{2}}{\partial \mathcal{E}_{b}} \frac{\partial \rho}{\partial \mathcal{E}_{c}}\right.\right. \\
& \left.+\frac{\partial^{3} f^{X C}}{\partial \rho^{2} \partial|\nabla \rho|^{2}} \frac{\partial \rho}{\partial \mathcal{E}_{b}} \frac{\partial \rho}{\partial \mathcal{E}_{c}}+\frac{\partial^{2} f^{X C}}{\partial \rho \partial|\nabla \rho|^{2}} \frac{\partial^{2} \rho}{\partial \mathcal{E}_{b} \partial \mathcal{E}_{c}}\right) \nabla \rho \\
& \left.+P_{b, c} \frac{\partial^{2} f^{X C}}{\partial\left(|\nabla \rho|^{2}\right)^{2}} \frac{\partial|\nabla \rho|^{2}}{\partial \mathcal{E}_{c}} \frac{\partial \nabla \rho}{\partial \mathcal{E}_{b}}+P_{b, c} \frac{\partial^{2} f^{X C}}{\partial \rho \partial|\nabla \rho|^{2}} \frac{\partial \rho}{\partial \mathcal{E}_{c}} \frac{\partial \nabla \rho}{\partial \mathcal{E}_{b}}+\frac{\partial f^{X C}}{\partial|\nabla \rho|^{2}} \frac{\partial^{2} \nabla \rho}{\partial \mathcal{E}_{b} \partial \mathcal{E}_{c}}\right] \cdot \nabla \rho^{\left(\mathcal{R}_{a}^{A}\right)} \\
& +P_{b, c}\left[\frac{\partial^{2} f^{X C}}{\partial \rho^{2}} \frac{\partial \rho}{\partial \mathcal{E}_{b}}+\frac{\partial^{2} f^{X C}}{\partial \rho \partial|\nabla \rho|^{2}} \frac{\partial|\nabla \rho|^{2}}{\partial \mathcal{E}_{b}}\right] \frac{\partial \rho^{\left(\mathcal{R}_{a}^{A}\right)}}{\partial \mathcal{E}_{c}} \\
& +2 P_{b, c}\left[\left(\frac{\partial^{2} f^{X C}}{\partial\left(|\nabla \rho|^{2}\right)^{2}} \frac{\partial|\nabla \rho|^{2}}{\partial \mathcal{E}_{b}}+\frac{\partial^{2} f^{X C}}{\partial \rho \partial|\nabla \rho|^{2}} \frac{\partial \rho}{\partial \mathcal{E}_{b}}\right) \nabla \rho+\frac{\partial f^{X C}}{\partial|\nabla \rho|^{2}} \frac{\partial \nabla \rho}{\partial \mathcal{E}_{b}}\right] \cdot \frac{\partial \nabla \rho^{\left(\mathcal{R}_{a}^{A}\right)}}{\partial \mathcal{E}_{c}} \\
& \left.+\frac{\partial f^{X C}}{\partial \rho} \frac{\partial^{2} \rho^{\left(\mathcal{R}_{a}^{A}\right)}}{\partial \mathcal{E}_{b} \partial \mathcal{E}_{c}}+2 \frac{\partial f^{X C}}{\partial|\nabla \rho|^{2}} \nabla \rho \cdot \frac{\partial^{2} \nabla \rho^{\left(\mathcal{R}_{a}^{A}\right)}}{\partial \mathcal{E}_{b} \partial \mathcal{E}_{c}}\right\}_{\mathbf{r}_{i}} \\
& +\sum_{i} \frac{\partial w_{i}}{\partial \mathcal{R}_{a}^{A}}\left\{\frac{\partial^{2} f^{X C}}{\partial \rho^{2}} \frac{\partial \rho}{\partial \mathcal{E}_{b}} \frac{\partial \rho}{\partial \mathcal{E}_{c}}+P_{b, c} \frac{\partial^{2} f^{X C}}{\partial \rho \partial|\nabla \rho|^{2}} \frac{\partial|\nabla \rho|^{2}}{\partial \mathcal{E}_{b}} \frac{\partial \rho}{\partial \mathcal{E}_{c}}+\frac{\partial^{2} f^{X C}}{\partial\left(|\nabla \rho|^{2}\right)^{2}} \frac{\partial|\nabla \rho|^{2}}{\partial \mathcal{E}_{b}} \frac{\partial|\nabla \rho|^{2}}{\partial \mathcal{E}_{c}}\right. \\
& \left.+\frac{\partial f^{X C}}{\partial \rho} \frac{\partial^{2} \rho}{\partial \mathcal{E}_{b} \partial \mathcal{E}_{c}}+\frac{\partial f^{X C}}{\partial|\nabla \rho|^{2}} \frac{\partial^{2}\left(|\nabla \rho|^{2}\right)^{2}}{\partial \mathcal{E}_{b} \partial \mathcal{E}_{c}}\right\}_{\mathbf{r}_{i}} .
\end{aligned}
$$

The XC functional derivatives are obtained through the XCFUN library written by Ekström ${ }^{53,54}$ using automatic differentiation for arbitrary-order derivatives. This library has been interfaced to the CRYSTAL program. ${ }^{42}$

\section{CONCLUSIONS}

We have developed a novel method for the fully analytical calculation of Raman intensities at the Hartree-Fock and DFT level. Our treatment, which has been implemented in the CRYSTAL code, involves solving only a single second-order self-consistent equation for each polarizability component.
This remains true regardless of the number of atoms in the unit cell. Numerical differentiation with respect to atomic coordinates and with respect to wave vectors is entirely avoided.

Despite an involved derivation the final expressions are simple and computationally optimal. This allows for the easy simulation of Raman spectra for crystalline systems periodic in one, two, and three dimensions.

In Paper II of this article, ${ }^{34}$ we present results computed for different crystalline systems, in order to validate the method, including comparison with experiment, as well as verify its numerical stability and efficiency. As shown there, Raman spectra of systems with more than a hundred 
atoms in the unit cell, such as UiO-66 Metal Organic Framework, can be simulated with a reasonable computational effort.

The treatment that has been presented can be extended to other properties such as analytical evaluation of hyperRaman intensities, vibrational circular dichroism, and vibrational nonlinear optical properties. We intend to carry out some of these further developments in the future.

The treatment presented here is for the purpose of calculating Raman spectra and, hence, will be applied in Paper $\mathrm{II}^{34}$ to $\vec{k}=0$ vibrations, i.e., at the $\Gamma$ point. No such restriction has been applied to the displacements $\mathcal{R}_{a}^{A}$.

\section{APPENDIX: DERIVATIVES OF THE $\Xi$ MATRICES}

In this Appendix, we report the detailed derivations that were omitted in the main body of the article for the sake of enhancing the readability of the paper. Thus, for the differentiation of $\Xi_{b}(\vec{k})$ with respect to a field $\mathcal{E}_{c}$, i.e., Eq. (36), we have

$$
\begin{aligned}
\iota \Xi_{b}^{\left(\mathcal{E}_{c}\right)}(\vec{k}) & =\iota S(\vec{k})\left[\left(\frac{\partial}{\partial k_{b}} C^{(0)}(\vec{k}) U^{\left(\mathcal{E}_{c}\right)}(\vec{k})\right) C^{(0) \dagger}(\vec{k})+\left(\frac{\partial}{\partial k_{b}} C^{(0)}(\vec{k})\right) U^{\left(\mathcal{E}_{c}\right) \dagger}(\vec{k}) C^{(0) \dagger}(\vec{k})\right] S(\vec{k}) \\
& =\iota S(\vec{k}) C^{(0)}(\vec{k})\left(Q_{b}(\vec{k}) U^{\left(\mathcal{E}_{c}\right)}(\vec{k})+\frac{\partial U^{\left(\mathcal{E}_{c}\right)}(\vec{k})}{\partial k_{b}}+Q_{b}(\vec{k}) U^{\left(\mathcal{E}_{c}\right) \dagger}(\vec{k})\right) C^{(0) \dagger}(\vec{k}) S(\vec{k}) \\
& =\iota S(\vec{k}) C^{(0)}(\vec{k})\left(Q_{b}(\vec{k}) U^{\left(\mathcal{E}_{c}\right)}(\vec{k})+\frac{\partial U^{\left(\mathcal{E}_{c}\right)}(\vec{k})}{\partial k_{b}}-Q_{b}(\vec{k}) U^{\left(\mathcal{E}_{c}\right)}(\vec{k})\right) C^{(0) \dagger}(\vec{k}) S(\vec{k}) \\
& =\iota S(\vec{k}) C^{(0)}(\vec{k}) \frac{\partial U^{\left(\mathcal{E}_{c}\right)}(\vec{k})}{\partial k_{b}} C^{(0) \dagger}(\vec{k}) S(\vec{k}),
\end{aligned}
$$

which follows from the fact that $U^{\left(\mathcal{E}_{c}\right)}(\vec{k})$ is anti-hermitian. Then, for the derivative with respect to an atomic displacement $\mathcal{R}_{a}^{A}$, i.e., Eq. (61), the following provides a step-by-step derivation:

$$
\begin{aligned}
& \Xi_{b}^{\left(\mathcal{R}_{a}^{A}\right)}(\vec{k})=S^{\left(\mathcal{R}_{a}^{A}\right)}(\vec{k})\left[\frac{\partial C^{(0)}(\vec{k})}{\partial k_{b}}\right] C^{(0) \dagger}(\vec{k}) S(\vec{k})+S(\vec{k})\left[\frac{\partial C^{\left(\mathcal{R}_{a}^{A}\right)}(\vec{k})}{\partial k_{b}}\right] C^{(0) \dagger}(\vec{k}) S(\vec{k}) \\
& +S(\vec{k})\left[\frac{\partial C^{(0)}(\vec{k})}{\partial k_{b}}\right] C^{\left(\mathcal{R}_{a}^{A}\right) \dagger}(\vec{k}) S(\vec{k})+S(\vec{k})\left[\frac{\partial C^{(0)}(\vec{k})}{\partial k_{b}}\right] C^{(0) \dagger}(\vec{k}) S^{\left(\mathcal{R}_{a}^{A}\right)}(\vec{k}) \\
& =S^{\left(\mathcal{R}_{a}^{A}\right)}(\vec{k}) C^{(0)}(\vec{k}) Q_{b}(\vec{k}) C^{(0) \dagger}(\vec{k}) S(\vec{k})+S(\vec{k}) C^{(0)}(\vec{k}) Q_{b}(\vec{k}) C^{(0) \dagger}(\vec{k}) S^{\left(\mathcal{R}_{a}^{A}\right)}(\vec{k}) \\
& +S(\vec{k}) C^{(0)}(\vec{k})\left[Q_{b}(\vec{k}) U^{\left(\mathcal{R}_{a}^{A}\right)}(\vec{k})+\frac{\partial U^{\left(\mathcal{R}_{a}^{A}\right)}(\vec{k})}{\partial k_{b}}+Q_{b}(\vec{k}) U^{\left(\mathcal{R}_{a}^{A}\right) \dagger}(\vec{k})\right] C^{(0) \dagger}(\vec{k}) S(\vec{k}) \\
& =S^{\left(\mathcal{R}_{a}^{A}\right)}(\vec{k}) C^{(0)}(\vec{k}) Q_{b}(\vec{k}) C^{(0) \dagger}(\vec{k}) S(\vec{k})+S(\vec{k}) C^{(0)}(\vec{k}) Q_{b}(\vec{k}) C^{(0) \dagger}(\vec{k}) S^{\left(\mathcal{R}_{a}^{A}\right)}(\vec{k}) \\
& +S(\vec{k}) C^{(0)}(\vec{k})\left[\frac{\partial U^{\left(\mathcal{R}_{a}^{A}\right)}(\vec{k})}{\partial k_{b}}-Q_{b}(\vec{k}) C^{(0) \dagger}(\vec{k}) S^{\left(\mathcal{R}_{a}^{A}\right)}(\vec{k}) C^{(0)}(\vec{k})\right] C^{(0) \dagger}(\vec{k}) S(\vec{k}) .
\end{aligned}
$$


Here we have used Eqs. (21), (44), (47) and, in the last step, Eq. (46). Finally, combining the expression of $\Xi_{b}^{\left(\mathcal{R}_{a}^{A}, \mathcal{E}_{c}\right)}(\vec{k})$ resulting from Eq. (67) with the unperturbed density matrix we obtain the result of Eq. (68),

$$
\begin{aligned}
& \sum_{\vec{k}} \operatorname{Tr}\left(\iota \Xi_{b}^{\left(\mathcal{R}_{a}^{A}, \mathcal{E}_{c}\right)}(\vec{k}) D^{(0)}(\vec{k})\right) \\
& =\imath \sum_{\vec{k}} \operatorname{Tr}\left(C^{(0) \dagger}(\vec{k}) S^{\left(\mathcal{R}_{a}^{A}\right)}(\vec{k}) C^{(0)}(\vec{k})\left[\frac{\partial U^{\left(\mathcal{E}_{c}\right)}(\vec{k})}{\partial k_{b}} n+n \frac{\partial U^{\left(\mathcal{E}_{c}\right)}(\vec{k})}{\partial k_{b}}\right]\right. \\
& \left.+\left[U^{\left(\mathcal{R}_{a}^{A}\right)}(\vec{k}) \frac{\partial U^{\left(\mathcal{E}_{c}\right)}(\vec{k})}{\partial k_{b}}+\left(\frac{\partial U^{\left(\mathcal{E}_{c}\right)}(\vec{k})}{\partial k_{b}}\right)^{\left(\mathcal{R}_{a}^{A}\right)}+\frac{\partial U^{\left(\mathcal{E}_{c}\right)}(\vec{k})}{\partial k_{b}} U^{\left(\mathcal{R}_{a}^{A}\right) \dagger}(\vec{k})\right] n\right) \\
& =\iota \sum_{\vec{k}} \operatorname{Tr}\left(C^{(0) \dagger}(\vec{k}) S^{\left(\mathcal{R}_{a}^{A}\right)}(\vec{k}) C^{(0)}(\vec{k})\left[\frac{\partial U^{\left(\mathcal{E}_{c}\right)}(\vec{k})}{\partial k_{b}} n+n \frac{\partial U^{\left(\mathcal{E}_{c}\right)}(\vec{k})}{\partial k_{b}}\right]+U^{\left(\mathcal{R}_{a}^{A}\right)}(\vec{k}) \frac{\partial U^{\left(\mathcal{E}_{c}\right)}(\vec{k})}{\partial k_{b}} n\right. \\
& \left.-\frac{\partial U^{\left(\mathcal{E}_{c}\right)}(\vec{k})}{\partial k_{b}}\left[U^{\left(\mathcal{R}_{a}^{A}\right)}(\vec{k})+C^{(0) \dagger}(\vec{k}) S^{\left(\mathcal{R}_{a}^{A}\right)}(\vec{k}) C^{(0)}(\vec{k})\right] n\right) \\
& =\imath \sum_{\vec{k}} \operatorname{Tr}\left(U^{\left(\mathcal{R}_{a}^{A}\right)}(\vec{k})\left[\frac{\partial U^{\left(\mathcal{E}_{c}\right)}(\vec{k})}{\partial k_{b}} n-n \frac{\partial U^{\left(\mathcal{E}_{c}\right)}(\vec{k})}{\partial k_{b}}\right]+C^{(0) \dagger}(\vec{k}) S^{\left(\mathcal{R}_{a}^{A}\right)}(\vec{k}) C^{(0)}(\vec{k}) \frac{\partial U^{\left(\mathcal{E}_{c}\right)}(\vec{k})}{\partial k_{b}} n\right)
\end{aligned}
$$

since $\partial U^{\left(\mathcal{E}_{c}\right)}(\vec{k}) / \partial k_{b}$ is block off-diagonal at all $\mathcal{R}_{a}^{A}$.

${ }^{1}$ R. L. McCreery, in Handbook of Vibrational Spectroscopy, edited by J. Chalmers and P. Griffiths (John Wiley \& Sons, Chichester, 2002), pp. 920932.

${ }^{2}$ G. Placzek, Handbuch der Radiologie (Akademische Verlagsgeselschft, Leipzig, 1934), Vol. 6, p. 208.

${ }^{3}$ R. D. Amos, Chem. Phys. Lett. 124, 376 (1986).

${ }^{4}$ M. J. Frisch, Y. Yamaguchi, J. F. Gaw, H. F. Schaefer III, and J. S. Binkley, J. Chem. Phys. 84, 531 (1986).

${ }^{5}$ P. Otto, Phys. Rev. B 45, 10876 (1992).

${ }^{6}$ B. Champagne, J. G. Fripiat, and J. André, J. Chem. Phys. 96, 8330 (1992).

${ }^{7}$ P. Otto, F. L. Gu, and J. Ladik, J. Chem. Phys. 110, 2717 (1999).

${ }^{8}$ B. Kirtman, F. L. Gu, and D. M. Bishop, J. Chem. Phys. 113, 1294 (2000).

${ }^{9}$ F. L. Gu, D. M. Bishop, and B. Kirtman, J. Chem. Phys. 115, 10548 (2001).

${ }^{10}$ K. N. Kudin and G. Scuseria, J. Chem. Phys. 113, 7779 (2000).

${ }^{11}$ D. M. Bishop, F. L. Gu, and B. Kirtman, J. Chem. Phys. 114, 7633 (2001).

${ }^{12}$ P. Otto, A. Martinez, A. Czaja, and J. Ladik, J. Chem. Phys. 117, 1908 (2002).

${ }^{13}$ X. Gonze et al., Comput. Phys. Commun. 180, 2582 (2009).

${ }^{14}$ P. Giannozzi et al., J. Phys.: Condens. Matter 21, 395502 (2009).

${ }^{15}$ X. Gonze, Phys. Rev. B 55, 10337 (1997).

${ }^{16}$ S. Baroni, S. de Gironcoli, A. dal Corso, and P. Giannozzi, Rev. Mod. Phys. 73, 515 (2001).

${ }^{17}$ L. Maschio, B. Kirtman, R. Orlando, and M. Rérat, J. Chem. Phys. 137, 204113 (2012).

${ }^{18}$ M. Ferrero, M. Rérat, B. Kirtman, and R. Dovesi, J. Chem. Phys. 129, 244110 (2008).

${ }^{19}$ M. Ferrero, M. Rérat, R. Orlando, and R. Dovesi, J. Comput. Chem. 29, 1450 (2008).

${ }^{20}$ M. Ferrero, M. Rérat, R. Orlando, and R. Dovesi, J. Chem. Phys. 128, 014110 (2008).

${ }^{21}$ R. Dovesi, V. R. Saunders, C. Roetti, R. Orlando, C. M. Zicovich-Wilson, F. Pascale, K. Doll, N. M. Harrison, B. Civalleri, I. J. Bush et al., CRYSTAL09 User's Manual (Università di Torino, Torino, 2009).
${ }^{22}$ R. Dovesi, R. Orlando, B. Civalleri, C. Roetti, V. Saunders, and C. Zicovich-Wilson, Z. Kristallogr. 220, 571 (2005).

${ }^{23}$ S. Baroni and R. Resta, Phys. Rev. B 33, 5969 (1986).

${ }^{24}$ P. Umari, A. Pasquarello, and A. D. Corso, Phys. Rev. B 63, 094305 (2001).

${ }^{25}$ E. Spanó and M. Bernasconi, Phys. Rev. B 71, 174301 (2005).

${ }^{26}$ V. Milman, K. Refson, S. Clark, C. Pickard, J. Yates, S.-P. Gao, P. Hasnip, M. Probert, A. Perlov, and M. Segall, J. Mol. Struct.: THEOCHEM 954, 22 (2010).

${ }^{27}$ K. Miwa, Phys. Rev. B 84, 094304 (2011).

${ }^{28}$ M. Lazzeri and F. Mauri, Phys. Rev. Lett. 90, 036401 (2003).

${ }^{29}$ M. Veithen, X. Gonze, and P. Ghosez, Phys. Rev. B 71, 125107 (2005).

${ }^{30}$ G. Deinzer and D. Strauch, Phys. Rev. B 66, 100301(R) (2002).

${ }^{31}$ L. Maschio, B. Kirtman, M. Rérat, R. Orlando, and R. Dovesi, "Comment on " Ab initio analytical infrared intensities for periodic systems through a coupled perturbed Hartree-Fock/Kohn-Sham method,"' J. Chem. Phys. (to be published).

${ }^{32}$ R. Bast, U. Ekstrom, B. Gao, T. Helgaker, K. Ruud, and A. J. Thorvaldsen, Phys. Chem. Chem. Phys. 13, 2627 (2011).

${ }^{33}$ O. Quinet and B. Champagne, J. Chem. Phys. 115, 6293 (2001).

${ }^{34}$ L. Maschio, B. Kirtman, M. Rérat, R. Orlando, and R. Dovesi, J. Chem. Phys. 139, 164102 (2013).

${ }^{35}$ V. Saunders, C. Freyria-Fava, R. Dovesi, L. Salasco, and C. Roetti, Mol. Phys. 77, 629 (1992).

${ }^{36}$ K. Doll, N. M. Harrison, and V. R. Saunders, Int. J. Quantum Chem. 82, 1 (2001).

${ }^{37}$ J. Delhalle, J. M. André, C. Demanet, and J. L. Brédas, Chem. Phys. Lett. 54, 186 (1978).

${ }^{38}$ V. Saunders, C. Freyria-Fava, R. Dovesi, and C. Roetti, Comput. Phys. Commun. 84, 156 (1994).

${ }^{39}$ D. E. Parry, Surf. Sci. 49, 433 (1975).

${ }^{40}$ D. M. Heyes, M. Barber, and J. H. R. Clarke, J. Chem. Soc. Faraday Trans. II 73, 1485 (1977).

${ }^{41}$ P. P. Ewald, Ann. Phys. (Leipzig) 64, 253 (1921).

${ }^{42}$ R. Orlando, V. Lacivita, R. Bast, and K. Ruud, J. Chem. Phys. 132, 244106 (2010).

${ }^{43}$ K. Kudin, R. Car, and R. Resta, J. Chem. Phys. 126, 234101 (2007). 
${ }^{44}$ A. Izmaylov and G. Scuseria, Phys. Rev. B 77, 165131 (2008).

${ }^{45}$ M. Springborg and B. Kirtman, Phys. Rev. B 77, 045102 (2008).

${ }^{46} \mathrm{P}$. Pulay, Analytical Derivative Methods in Quantum Chemistry (John Wiley \& Sons, Inc., 2007), pp. 241-286.

${ }^{47}$ A. Szabo and N. S. Ostlund, Modern Quantum Chemistry (Dover Publications, 1989).

${ }^{48}$ There can be a phase difference between the crystal orbital coefficients on opposite sides of the Brillouin zone. Such a phase difference, if it exists, will alter the dipole moment but not the linear polarizability as shown in Ref. 11. Hence, in the calculation of Raman intensities we systematically ignore this possibility.
${ }^{49}$ At the present status symmetry has not been fully implemented for the CPHF procedure. We believe that when symmetry will be fully exploited this statement will become stronger for crystals with high symmetry.

${ }^{50}$ J. A. Pople, P. M. W. Gill, and B. G. Johnson, Chem. Phys. Lett. 199, 557 (1992).

${ }^{51}$ A. D. Becke, J. Chem. Phys. 88, 2547 (1988).

${ }^{52}$ M. Towler, A. Zupan, and M. Causà, Comput. Phys. Commun. 98, 181 (1996).

${ }^{53}$ U. Ekström, Xcfun library (2010), see http://www.admol.org/xcfun.

${ }^{54}$ U. Ekström, L. Visscher, R. Bast, A. J. Thorvaldsen, and K. Ruud, J. Chem. Theory Comput. 6, 1971 (2010). 